\title{
The influence of protecting groups on the diastereoselectivity of catalytic heterogeneous hydrogenation of Baylis-Hillman adducts
}

\author{
Fernando Coelho, ${ }^{* a}$ Wanda P. Almeida, ${ }^{\text {b }}$ Cristiano R. Mateus, ${ }^{\mathrm{a}}$ Leonardo D. Furtado, \\ and José Carlos F. Gouveia ${ }^{\text {a }}$ \\ ${ }^{a}$ DQO/IQ - UNICAMP- PO Box 6154 - 13084-917 - Campinas, S - Brazil \\ ${ }^{b}$ Curso de Pós-Graduação em Biologia Celular e Estrutural - IB/UNICAMP _ \\ PO Box 6109 - 13084-971 - Campinas, SP - Brazil . \\ E-mail: coelho@iqm.unicamp.br; wandaalmeida@terra.com.br
}

This article is dedicated to Prof. Edmundo Ruveda on occasion of his $70^{\text {th }}$ anniversary

(received 30 Aug 03; accepted 15 Oct 03; published on the web 22 Oct 03)

\begin{abstract}
In this communication we describe our recent results from a study on catalytic heterogeneous hydrogenation reactions of Baylis-Hillman adducts. Depending on the protecting groups used on the secondary hydroxyl group of these adducts, it is possible to obtain a high degree of diastereoselection. For silylated Baylis-Hillman adducts a high syn diastereoselectivity has been obtained. However, when secondary Baylis-Hillman hydroxyl groups were unprotected or protected as acetate a moderate anti selectivity was attained. The adducts protected as methyl ether gave poor syn diastereoselectivity.
\end{abstract}

Keywords: Baylis-Hillman, diastereoselectivity, heterogeneous hydrogenation

\section{Introduction}

The Baylis-Hillman reaction is a simple and straightforward alternative to generate a new C-C $\sigma$ bond. In the last few years this reaction has attracted the attention of many organic chemists, mainly due to the great synthetic potentiality of the $\alpha$-methylene- $\beta$-hydroxy compounds (Figure 1) provided by it, associated with its operational simplicity (no special experimental conditions such as low temperature, anhydrous solvent or inert atmosphere are required). This reaction may be broadly defined as a coupling between the $\alpha$-position of activated alkenes and an electrondeficient $\mathrm{sp}^{2}$ carbon atom, for instance an aldehyde carbonyl group. The reaction is usually catalyzed by DABCO (1,4-diazabicyclo [2.2.2.] octane) or a phosphine. ${ }^{1}$ 


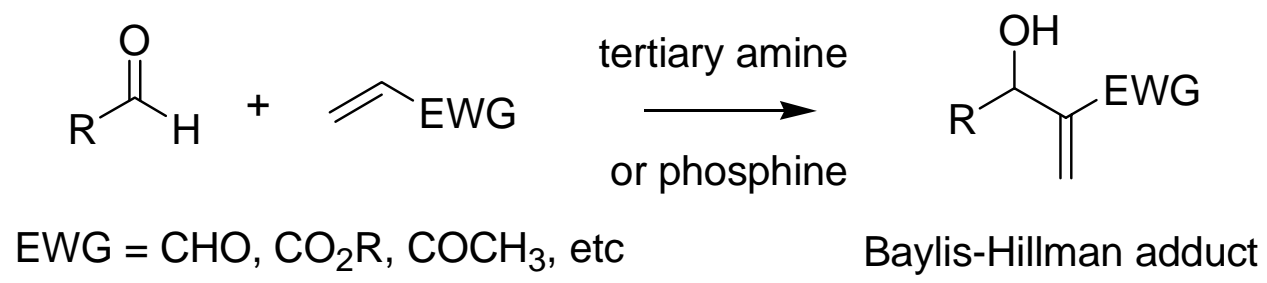

Figure 1. Baylis-Hillman reaction.

The major drawbacks from this reaction are its relative slowness (typically one to 4 weeks to be completed) associated with very low chemical yields (5-10\%). To solve this problem various modifications of the experimental protocol have been proposed, e.g., the use of microwaves, ${ }^{2}$ salts and metals ${ }^{3}$, ionic liquids ${ }^{4}$, an aqueous medium ${ }^{5}$ or the use of a Lewis acid, either as catalyst or as promotor. ${ }^{6}$ We have also demonstrated that the use of ultrasound dramatically accelerates the rate of this reaction. ${ }^{7}$

Recently, we were interested in using a hydrogenation reaction of a Baylis-Hillman adduct as a key step for the total synthesis of a natural pheromone. ${ }^{8}$ To our surprise, the literature contains several papers ${ }^{9-15}$ reporting the utilization of homogeneous catalytic hydrogenation of BaylisHillman adducts. Depending on the experimental conditions this hydrogenation reaction provides the anti diastereoisomers, with a high degree of diastereoselectivity and the syn diastereoisomer with moderate to excellent diastereoselectivity. ${ }^{15}$ In contrast there is only one report ${ }^{16}$ concerning the use of a heterogeneous catalyst, where no diastereoselectivity has been observed.

In a two preliminary studies on the heterogeneous catalytic hydrogenation of Baylis-Hillman adducts derived from aromatic and aliphatic aldehydes, ${ }^{8,17}$ we demonstrated that it was possible to obtain the syn isomer with a high degree of diastereoselectivity. The elevated degree of syn diastereoselection seems to be directly related to the presence of a protecting group on the Baylis-Hllman secondary hydroxyl group. These observations have been corroborated by the fact that it was possible to obtain poor to moderate anti diastereoselectivity if the Baylis-Hillman adduct were hydrogenated without protection of the secondary hydroxyl group (Scheme 1).

More recently, based on our preliminary results, Bouzide demonstrated that the utilisation of a chelating salt, such as $\mathrm{MgBr}_{2}$, could also dramatically improve the syn diastereoselectivity. ${ }^{18}$ Our previous results encouraged us to undertaken an additional study focused on determining the real influence of the protecting group on the diastereoselectivity of the heterogeneous catalytic hydrogenation with Baylis-Hillman adducts originating from both aliphatic and aromatic aldehydes. In this comunication we disclose the results we have obtained with this new study. 


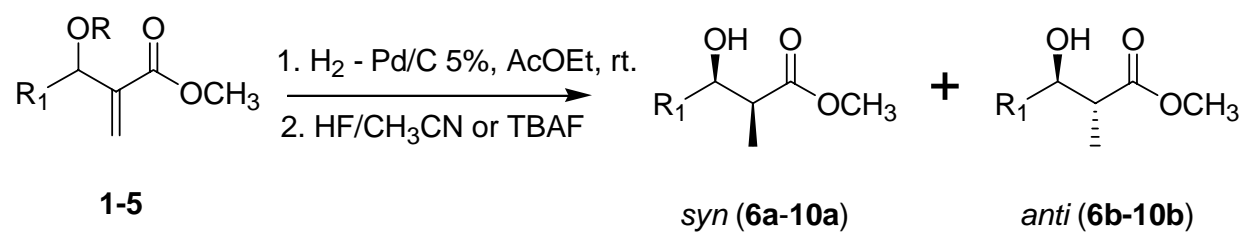

Baylis-Hillman adduct

$\mathrm{R}=\mathrm{H}$ or $\mathrm{TBS}$

6a/b, $\mathrm{R}_{1}=\mathrm{Ph} ; \mathbf{7 a} / \mathbf{b}, \mathrm{R}_{1}=4-\mathrm{OMePh} ; \mathbf{8} \mathbf{a} / \mathbf{b}, \mathrm{R}_{1}=4-\mathrm{ClPh}$

$9 \mathbf{a} / \mathbf{b}, \mathrm{R}_{1}=3,4-\mathrm{OCH}_{2} \mathrm{O}-\mathrm{Ph} ; \mathbf{1 0 a} / \mathbf{b}, \mathrm{R}_{1}=4-\mathrm{NO}_{2} \mathrm{Ph}$

\begin{tabular}{ccc}
\hline $\mathrm{R}_{1}$ & Syn : anti ratio $(\mathrm{R}=\mathrm{H})$ & Syn : anti ratio $(\mathrm{R}=\mathrm{TBS})$ \\
\hline 1, $\mathrm{Ph}$ & $1: 1$ & $10: 1$ \\
2, 4-OCH $\mathrm{Ph}$ & $1: 3$ & $19: 1$ \\
3, 4-Cl-Ph & $1: 2$ & $5: 1$ \\
4, 3,4- $-\mathrm{OCH}_{2} \mathrm{O}-\mathrm{Ph}$ & $1: 3$ & $10: 1$ \\
5, 4- $\mathrm{NO}_{2} \mathrm{Ph}$ & $1: 1$ & $16: 1$ \\
\hline
\end{tabular}

Scheme 1. Diastereoselective heterogeneous hydrogenation of Baylis-Hillman adduct.

\section{Results and Discussion}

In order to evaluate the influence of the protecting groups on the diastereoselectivity of the heterogeneous catalytic hydrogenation we have prepared several Baylis-Hillman adducts from aromatic and aliphatic aldehydes, using a method previously described by us. ${ }^{7}$ The BaylisHillman reaction between an aldehyde (aromatic or aliphatic) with methyl acrylate (with or without any additional solvent) in the presence of ultrasound radiation furnished the adducts (19) in good to very good chemical yields (Scheme 2). ${ }^{7 \mathrm{~b}, 8}$

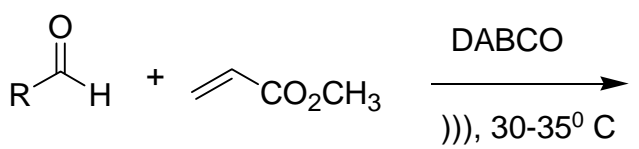<smiles>[R]C(O)C(=C)C(=O)OC</smiles>

1, $\mathrm{R}=\mathrm{Ph} ; 2, \mathrm{R}=4-\mathrm{OCH}_{3} \mathrm{Ph}$; 3, $\mathrm{R}=4-\mathrm{ClPh} ; 4,3,4-\mathrm{OCH}_{2} \mathrm{O}-$ $\mathrm{Ph} ; 5, \mathrm{R}=4-\mathrm{NO}_{2} \mathrm{Ph} ; 11, \mathrm{R}=$ methyl 12, R= ethyl; 13, R=npropyl; 14, R= $n$-butyl

Scheme 2. Baylis-Hillman reaction with aromatic and aliphatic aldehydes in the presence of ultrasound.

With the Baylis-Hillman adducts in our hands, the next step of our study was determining which hydroxyl protecting groups should be used. As our interest was centered in evaluating the influence of the spatial volume of protecting groups in the determination of the diastereoisomeric ratio during double bond hydrogenation, we have selected five different silylating reagents in 
which the substituents attached to the silicon have been gradually increased. Then, the BaylisHillman adducts (1-5) were treated with the chloride or the trifluoromethanesulfonate (triflate) of the following silylated protecting groups: trimethylsilyl (TMS), triethylsilyl (TES), tertbutyldimethylsilyl (TBDS), tri-isopropylsilyl (TIPS) and tert-butyldiphenylsilyl (TBDPS), in the presence of a base, to provide the corresponding silyl ethers. The results and chemical yields obtained in these protecting reactions are summarized in Table 1. We have chosen to start our study with Baylis-Hillman adducts from aromatic aldehydes only because they were less volatile and easier to manipulate than those from aliphatic aldehydes.

Table 1. Conditions and yields for the secondary hydroxyl protecting reactions

\begin{tabular}{|c|c|c|c|c|c|}
\hline Baylis-Hillman adducts & Silylating agents & Conditions & Time (h) & Product & Yields (\%) \\
\hline \multirow{5}{*}{1} & TMSCl & $\mathrm{NEt}_{3}, \mathrm{THF}, \mathrm{rt}$. & 6 & $15 a$ & 61 \\
\hline & TESCl & imidazole, DMF, rt. & 12 & $15 b$ & 70 \\
\hline & TIPSOTf & 2,6-lutidine, $\mathrm{CH}_{2} \mathrm{Cl}_{2}$, rt. & 1.5 & $15 c$ & 93 \\
\hline & TBDSCl & imidazole, DMF, rt. & 21 & 15d & 80 \\
\hline & TBDPSCl & imidazole, DMF, rt. & 12 & $15 e$ & 76 \\
\hline \multirow{5}{*}{$1 \mathrm{eO}^{-}$} & TMSCl & $\mathrm{NEt}_{3}, \mathrm{THF}, \mathrm{rt}$. & 6 & $16 \mathbf{a}$ & 56 \\
\hline & TESCl & imidazole, DMF, rt. & 12 & $16 b$ & 66 \\
\hline & TIPSOTf & 2,6-lutidine, $\mathrm{CH}_{2} \mathrm{Cl}_{2}$, rt. & 1 & $16 c$ & 83 \\
\hline & TBDSCl & imidazole, DMF, rt. & 21 & 16d & 88 \\
\hline & TBDPSCl & imidazole, DMF, rt. & 18 & $16 \mathrm{e}$ & 97 \\
\hline \multirow{5}{*}{$\mathrm{OH}$} & TMSCl & $\mathrm{NEt}_{3}, \mathrm{THF}$, rt. & 6 & $17 \mathbf{a}$ & 99 \\
\hline & TESCl & imidazole, DMF, rt. & 16 & $17 \mathbf{b}$ & 70 \\
\hline & TIPSOTf & 2,6-lutidine, $\mathrm{CH}_{2} \mathrm{Cl}_{2}$, rt. & 1 & 17c & 78 \\
\hline & TBDSCl & imidazole, DMF, rt. & 21 & 17d & 70 \\
\hline & TBDPSCl & imidazole, DMF, rt. & 12 & $17 \mathrm{e}$ & 72 \\
\hline \multirow{4}{*}{$\mathrm{OH}$} & TMSOTf & 2,6-lutidine, $\mathrm{CH}_{2} \mathrm{Cl}_{2}$, rt. & $1 / 2$ & $18 a$ & 70 \\
\hline & TESCl & imidazole, DMF, rt. & 12 & $18 b$ & 88 \\
\hline & TIPSOTf & 2,6-lutidine, $\mathrm{CH}_{2} \mathrm{Cl}_{2}$, rt. & 2 & $18 \mathrm{c}$ & 85 \\
\hline & TBDSCl & imidazole, DMF, rt. & 21 & 18d & 77 \\
\hline & TBDPSCl & imidazole, DMF, rt. & 12 & $18 \mathrm{e}$ & 93 \\
\hline \multirow{5}{*}{$\mathrm{O}_{2} \mathrm{~N}$} & TMSCl & $\mathrm{NEt}_{3}, \mathrm{THF}, \mathrm{rt}$. & 6 & 19a & 52 \\
\hline & TESCl & imidazole, DMF, rt. & 8 & $19 b$ & 97 \\
\hline & TIPSOTf & 2,6-lutidine, $\mathrm{CH}_{2} \mathrm{Cl}_{2}$, rt. & $1 / 2$ & 19c & 94 \\
\hline & TBDSOTf & 2,6-lutidine, $\mathrm{CH}_{2} \mathrm{Cl}_{2}$, rt & $1 / 2$ & 19d & 76 \\
\hline & TBDPSCl & imidazole, DMF, rt. & $-{ }^{b}$ & $-{ }^{b}$ & $-{ }^{b}$ \\
\hline
\end{tabular}


${ }^{a}$ Yields for isolated and purified products ${ }^{b}$ No protecting product was detected for this adduct under this experimental conditions.

The solution of silylated ethers 15a-e/19a-d (see Table 1) in ethyl acetate was treated with 5 $\mathrm{mol} \%$ of $\mathrm{Pd} / \mathrm{C} 5 \%$ under an atmosphere of hydrogen at room pressure to provide the corresponding reduced products as a mixture of diastereoisomers (Scheme 3 and Table 2).

This crude mixture was treated with a solution of $5 \%$ HF in acetonitrile or with TBAF in THF to provide the corresponding alcohols $\mathbf{6 a} / \mathbf{b}-\mathbf{- 1 0} \mathbf{a} / \mathbf{b}$ (Scheme 3).

Table 2. Diastereoselectivity for the hydrogenation of double bond of silylated Baylis-Hillman adducts

\begin{tabular}{|c|c|c|c|c|}
\hline Entry & Substrate & $\mathrm{SiR}_{3}$ & $\begin{array}{c}\text { Products ; } \\
\text { Hydrogenation/removal of } \\
\text { silyl groups }(\%)^{\mathrm{a}} \\
\end{array}$ & $\begin{array}{l}\text { syn : anti }{ }^{\mathrm{b}} \\
\quad \text { ratio }\end{array}$ \\
\hline 1 & $15 a$ & $-\mathrm{Si}\left(\mathrm{CH}_{3}\right)_{3}$ & $\mathbf{6 a} / \mathbf{b} ; 78$ & $5.6: 1$ \\
\hline 2 & $15 b$ & $-\mathrm{Si}(\mathrm{Et})_{3}$ & $7 \mathbf{a} / \mathbf{b} ; 70$ & $15: 1$ \\
\hline 3 & $15 \mathrm{c}$ & $-\mathrm{Si}\left[\left(\mathrm{CH}\left(\mathrm{CH}_{3}\right)_{2}\right)\right]_{3}$ & $\mathbf{8 a} / \mathbf{b} ; 92$ & $8: 1$ \\
\hline 4 & 15d & $-\mathrm{Si}(t-\mathrm{Bu})\left(\mathrm{CH}_{3}\right)_{2}$ & $\mathbf{9 a} / \mathbf{b} ; 92$ & $10: 1$ \\
\hline 5 & 15e & $-\mathrm{Si}(t-\mathrm{Bu})(\mathrm{Ph})_{2}$ & $\mathbf{1 0 a} / \mathbf{b} ; 85$ & $6: 1$ \\
\hline 6 & $16 a$ & $-\mathrm{Si}\left(\mathrm{CH}_{3}\right)_{3}$ & $\mathbf{6 a} / \mathbf{b} ; 82$ & $7: 1$ \\
\hline 7 & $16 b$ & $-\mathrm{Si}(\mathrm{Et})_{3}$ & $7 \mathbf{a} / \mathbf{b} ; 60$ & $19: 1$ \\
\hline 8 & $16 c$ & $-\mathrm{Si}\left[\left(\mathrm{CH}\left(\mathrm{CH}_{3}\right)_{2}\right)\right]_{3}$ & 8a/b ; 78 & $12: 1$ \\
\hline 9 & 16d & $-\mathrm{Si}(\mathrm{t}-\mathrm{Bu})\left(\mathrm{CH}_{3}\right)_{2}$ & $\mathbf{8 a} / \mathbf{b} ; 80$ & $19: 1$ \\
\hline 10 & $16 e$ & $-\mathrm{Si}(t-\mathrm{Bu})(\mathrm{Ph})_{2}$ & $\mathbf{1 0 a} / \mathbf{b} ; 78$ & $12: 1$ \\
\hline 11 & $17 \mathbf{a}$ & $-\mathrm{Si}\left(\mathrm{CH}_{3}\right)_{3}$ & $\mathbf{6 a} / \mathbf{b} ; 50$ & $5.2: 1$ \\
\hline 12 & $17 b$ & $-\mathrm{Si}(\mathrm{Et})_{3}$ & $7 \mathbf{a} / \mathbf{b} ; 60$ & $4.8: 1$ \\
\hline 13 & 17c & $-\mathrm{Si}\left[\left(\mathrm{CH}\left(\mathrm{CH}_{3}\right)_{2}\right)\right]_{3}$ & $\mathbf{8 a} / \mathbf{b} ; 87$ & $13: 1$ \\
\hline 14 & 17d & $-\mathrm{Si}(t-\mathrm{Bu})\left(\mathrm{CH}_{3}\right)_{2}$ & $\mathbf{9 a} / \mathbf{b} ; 85$ & $4.8: 1$ \\
\hline 15 & $17 \mathrm{e}$ & $-\mathrm{Si}(t-\mathrm{Bu})(\mathrm{Ph})_{2}$ & $\mathbf{1 0 a} / \mathbf{b} ; 68$ & $3.5: 1$ \\
\hline 16 & $18 \mathbf{a}$ & $-\mathrm{Si}\left(\mathrm{CH}_{3}\right)_{3}$ & $\mathbf{6 a} / \mathbf{b} ; 70$ & $3.5: 1$ \\
\hline 17 & $18 b$ & $-\mathrm{Si}(\mathrm{Et})_{3}$ & 7a/b ; 74 & $7.3: 1$ \\
\hline 18 & $18 c$ & $-\mathrm{Si}\left[\left(\mathrm{CH}\left(\mathrm{CH}_{3}\right)_{2}\right)\right]_{3}$ & $\mathbf{8 a} / \mathbf{b} ; 69$ & $9: 1$ \\
\hline 19 & 18d & $-\mathrm{Si}(t-\mathrm{Bu})\left(\mathrm{CH}_{3}\right)_{2}$ & $\mathbf{9 a} / \mathbf{b} ; 92$ & $10: 1$ \\
\hline 20 & $18 \mathrm{e}$ & $-\mathrm{Si}(\mathrm{t}-\mathrm{Bu})(\mathrm{Ph})_{2}$ & $\mathbf{1 0 a} / \mathbf{b} ; 75$ & $8: 1$ \\
\hline 21 & $19 a^{c}$ & $-\mathrm{Si}\left(\mathrm{CH}_{3}\right)_{3}$ & $\mathbf{6 a} / \mathbf{b} ; 83$ & $5.6: 1$ \\
\hline 22 & $19 b^{c}$ & $-\mathrm{Si}(\mathrm{Et})_{3}$ & $7 \mathbf{a} / \mathbf{b} ; 83$ & $7: 1$ \\
\hline 23 & $19 c^{c}$ & $-\mathrm{Si}\left[\left(\mathrm{CH}\left(\mathrm{CH}_{3}\right)_{2}\right)\right]_{3}$ & $\mathbf{8 a} / \mathbf{b} ; 69$ & $3.5: 1$ \\
\hline
\end{tabular}


24 $\mathbf{1 9 d}^{\mathrm{c}} \quad-\mathrm{Si}(t-\mathrm{Bu})\left(\mathrm{CH}_{3}\right)_{2}$

9a/b ; 82

$16: 1$

${ }^{\mathrm{a}}$ Yields for isolated and purified products. ${ }^{\mathrm{b}}$ The diastereoisomeric ratio was determined by GC (HP column) and measured by ${ }^{1} \mathrm{H}$ NMR. ${ }^{\circ}$ In the hydrogenation reaction of the nitro derivative, we have also detected the reduction of the nitro groups, which was transformed to the corresponding amine.

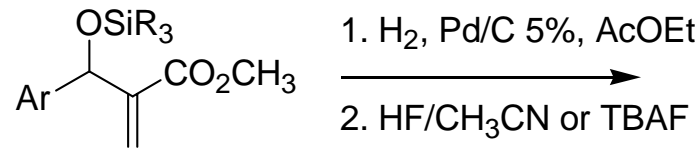

15a-e/19a-d<smiles>CC(=O)C(C)C(O)C(Br)Br</smiles>

syn<smiles>CC(=O)C(C)[C@H]([14CH3])O</smiles>

anti

$6 a / b-10 a / b$

Scheme 3. Heterogeneous catalytic hydrogenation reaction of silylated Baylis-Hillman adducts.

The relative stereochemistry (C2/C3) of the products was determined using ${ }^{1} \mathrm{H}$ NMR and ${ }^{13} \mathrm{C}$ NMR spectroscopy, the vicinal constant coupling $(J)$ for syn diastereoisomer varying between 5.9-6.32 $\mathrm{Hz}$ and 7-9 $\mathrm{Hz}$ for the anti isomer. ${ }^{18}$

The results obtained in the hydrogenation step are summarized in Table 2. The preliminary observations indicate that there was a dependence on the size of the substituents directly bonded to the silicon atom and the diastereoselectivity. Any silylated protecting groups led to the syn diastereoisomer as the major isomer. However, when huge silylated protecting groups such as triethylsilyl, tri-isopropylsilyl or tert-butyldimethyl silyl were used, it is possible to obtain the greatest proportion of the syn isomer. The minimized conformations for two silylated BaylisHillman adducts are shown in Figure 2.

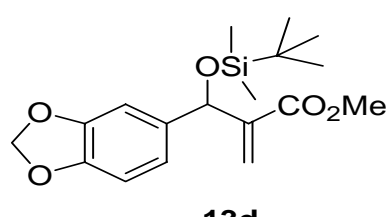

$13 d$

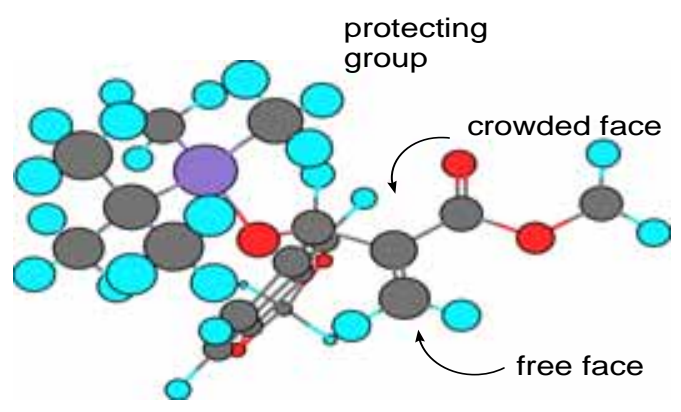



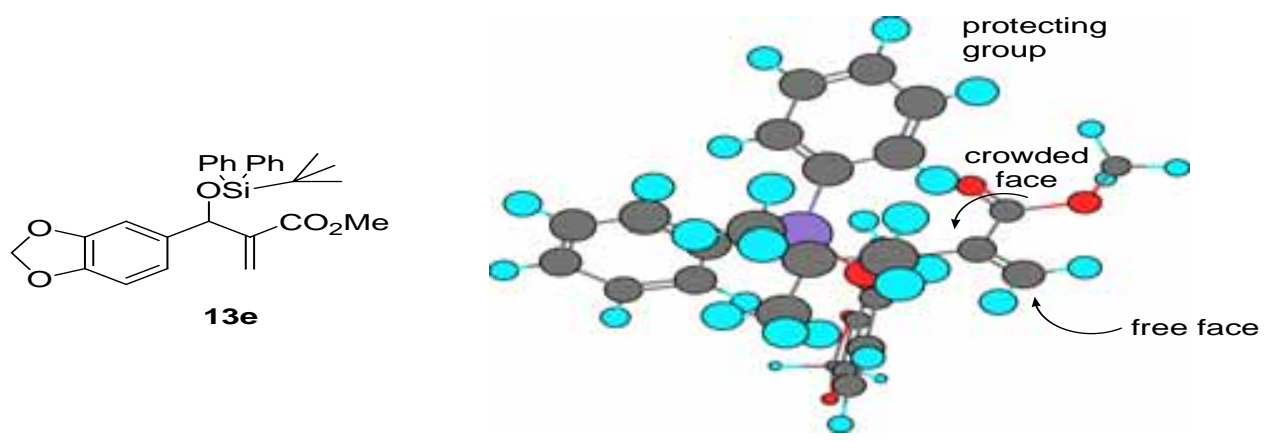

Figure 2. Minimized conformations of silylated Baylis-Hillman adducts.

A preliminary analysis pointed out that there was a difference between the faces of the double bond exerted by the protecting groups. The presence of a silyl group seems to be a sufficient condition to get a moderate to good degree of stereoselection in the heterogeneous hydrogenation reaction of Baylis-Hillman adducts. For silylated groups with large substituents on the silicon the diastereoselectivity seems to be better, however it is clear that there is a limit to the size of these substituents. For example , the syn diastereoselectivity for the tert-butyldiphenyl silyl groups (entries 5, 10, 15 and 20 of Table 2) is worse than that obtained for triethyl- (entries 2, 7, 12, 17 and 22 of Table 2), triisopropyl- (entries 3, 8, 13, 18 and 23 of Table 2) and tertbutyldimethylsilyl (entries 4, 9, 14, 19, 24 of Table 2) groups. Apparently, the diastereoselectivity was guided only by steric effects (see Figure 2). To evaluate the accuracy of these experimental observations we decided to test some additional protecting groups. Thus, we have acetylated and methylated the secondary hydroxyl group of some Baylis-Hillman adducts, which were submitted to experimental hydrogenation conditions similar to those described above.

Thus, the Baylis-Hillman adducts 1-5 were treated wih acetyl chloride in dicloromethane in the presence of triethylamine to give the corresponding acetates 20-24. To prepare the alkoxy derivatives, adducts 1-3 were treated with $\mathrm{Ag}_{2} \mathrm{O}$ and $\mathrm{CH}_{3} \mathrm{I}$ in dichloromethane to give the corresponding methyl ethers 25-27 (Scheme 4). ${ }^{19}$

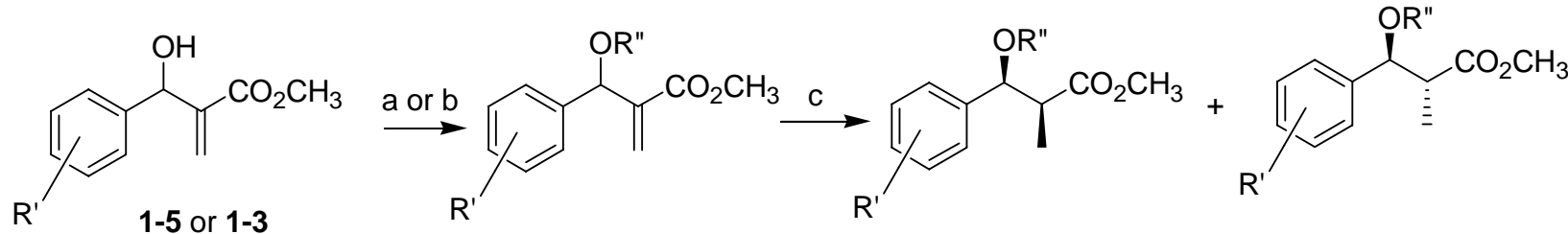

1, $\mathrm{R}^{\prime}=\mathrm{H} ; 2, \mathrm{R}=4-\mathrm{OCH}_{3}$

3, $\mathrm{R}^{\prime}=4-\mathrm{Cl} ; 4, \mathrm{R}^{\prime}=3,4-\mathrm{OCH}_{2} \mathrm{O}-$

5, $\mathrm{R}^{\prime}=4-\mathrm{NO}_{2}$
20-24, R"= $\mathrm{COCH}_{3}$

25-27, R"= $\mathrm{CH} 3$ 28a/b-32a/b, R"= $\mathrm{COCH}_{3}$

33a/b-35a/b, R" $=\mathrm{CH}_{3}$ 
Scheme 4. Reagents and conditions: a. $\mathrm{CH}_{3} \mathrm{COCl}, \mathrm{Et}_{3} \mathrm{~N}, \mathrm{CH}_{2} \mathrm{Cl}_{2}$, rt.; b. $\mathrm{Ag}_{2} \mathrm{O}, \mathrm{CH}_{3} \mathrm{I}, \mathrm{CH}_{2} \mathrm{Cl}_{2}$, rt.; c. Pd/C 5\%, AcOEt, rt.

Hydrogenation of both acetylated and methylated adducts gave a mixture of diastereoisomers with very poor anti diastereoselectivity for the first case and poor syn diastereoselectivity for the methyl ethers. The results are summarized in Table 3.

The chemical yields obtained, both for the protecting reactions and for the hydrogenation step, could be considered from moderate to very good. For the methylated adducts we have observed a discrete syn diastereoselectivity. Probably the spatial volume occupied by methyl groups is not enough to guide the hydrogen addition selectivity to only one of the two faces of the double bond. This could justify the low degree of diastereoselection obtained in the cases where the protecting group was methyl. On the other hand, the heterogeneous catalytic hydrogenation reaction of the acetylated Baylis-Hillman adducts led to a mixture in which an inversion of the distribution of diastereoisomers has occurred. In these cases, despite the very poor diastereoselectivity attained, the major isomer was anti.

From our point of view, these results could be based on an electronic effect. Alternatively, a configuration where the double bond is freed from the influence of the substituents could be also responsible for these results.

Table 3. Hydrogenation of the acetylated and methylated Baylis-Hillman adducts

\begin{tabular}{|c|c|c|c|c|}
\hline Entry & $\begin{array}{l}\text { Acetylated and Methylated Baylis- } \\
\text { Hillman adducts }\end{array}$ & $\begin{array}{c}\text { Protection Yield } \\
(\%)^{\mathrm{a}, \mathrm{b}}\end{array}$ & syn : anti ratio & $\begin{array}{l}\text { Hydrogenation } \\
(\%)^{\mathrm{a}}\end{array}$ \\
\hline 1 & 20, R'=H, R”=Ac & 82 & $\mathbf{2 8 a} / \mathbf{b} ; 1: 3$ & $65^{\mathrm{b}}$ \\
\hline 2 & 21, R'= $\mathrm{OCH}_{3}, \mathrm{R}^{\prime \prime}=\mathrm{Ac}$ & 78 & 29a/b; $1: 1$ & $70^{\mathrm{b}}$ \\
\hline 3 & 22, $\mathrm{R}^{\prime}=\mathrm{Cl}, \mathrm{R}{ }^{\prime}=\mathrm{Ac}$ & 80 & 30a/b; $1: 1.7$ & $63^{\mathrm{b}}$ \\
\hline 4 & 23, $\mathrm{R}^{\prime}=3,4-\mathrm{OCH}_{2} \mathrm{O}, \mathrm{R} "=\mathrm{Ac}$ & 89 & 31a/b; $1: 2.3$ & $60^{\mathrm{b}}$ \\
\hline 5 & 24, $\mathrm{R}^{\prime}=\mathrm{NO}_{2}, \mathrm{R}^{\prime}=\mathrm{Ac}$ & 76 & 32a/b; $1: 1.5$ & $78^{\mathrm{b}}$ \\
\hline 7 & $25, \mathrm{R}^{\prime}=\mathrm{H} ; \mathrm{R}^{\prime \prime}=\mathrm{CH}_{3}$ & 85 & 33a/b; $3: 1$ & 90 \\
\hline 8 & 26, R'= $\mathrm{OCH}_{3}, \mathrm{R}^{\prime \prime}=\mathrm{CH}_{3}$ & 78 & 34a/b; $2.1: 1$ & 92 \\
\hline 9 & 27, R'= Cl, R” $=\mathrm{CH}_{3}$ & 84 & 35a/b; $2.3: 1$ & 89 \\
\hline
\end{tabular}

${ }^{\mathrm{a}}$ Yields are for isolated and purified products. ${ }^{\mathrm{b}}$ For all cases we have detected hydrogenolysis reaction which ratio varied from 10 to $20 \%$.

At this point in the work it was clear to us that it was possible to obtain an elevated degree of syn diastereoselection if we protect the secondary hydroxyl group of the Baylis-Hillman adducts as a silylated ether (preferentially using TES, TIPS or TBDS groups). To check the generality of these effects on diastereoselectivity we decided to hydrogenate some additional Baylis-Hillman adducts from aliphatic aldehydes. Solutions of Baylis-Hillman adducts (11-14) in 
dimethylformamide were treated with tert-butyldimethylsilyl chloride in the presence of imidazole to provide the corresponding silyl ethers (36-39) in chemical yields varying from 77$80 \%$. We decided to choose tert-butyldimethylsilyl as the standard protecting only due to the ease of working with it.

The results obtained with the heterogeneous hydrogenation of this new set of protected Baylis-Hillman adducts are summarized in Table 4.

In all silylated esters (Table 2 and 4) the presence of the silyl group blocks the $R e$ face, directing the delivery of the hydrogen preferentially to the $S i$ face to yield the syn diastereoisomer (Scheme 5).

Table 4. Hydrogenation of silylated Baylis-Hillman adducts from aliphatic aldehydes

\begin{tabular}{|c|c|c|c|c|}
\hline Entry & Baylis-Hillman adducts & Product/SiR 3 & $\begin{array}{l}\text { Reduction } \\
\text { yield (\%) }\end{array}$ & $\begin{array}{c}\text { Product/Ratio } \\
\text { syn : } a n t^{\mathrm{b}}\end{array}$ \\
\hline 1 & 11 & 36, TBDS & 78 & 40a/b, $6: 1$ \\
\hline 2 & 12 & 37, TBDS & 82 & 41a/b, $16: 1$ \\
\hline 3 & 13 & 38, TBDS & 76 & 42a/b, $16: 1$ \\
\hline 4 & 14 & 39, TBDS & 76 & 43a/b, $17: 1$ \\
\hline
\end{tabular}

${ }^{a}$ Yields for isolated and purified products. ${ }^{\mathrm{b}}$ The diastereoisomeric ratio was determined by GC (HP column) and measured by ${ }^{1} \mathrm{H}$ NMR.

The stereochemical outcome of these hydrogenation reactions can be rationalized based on a conformation in which the alkyl substituent occupies the inside position and the carbon-oxygen bond is perpendicular to the $\pi$ bond. This reduces any destablizing interactions between the alkyl substituents and the carboxyl group (Scheme 5). ${ }^{20-21}$ 


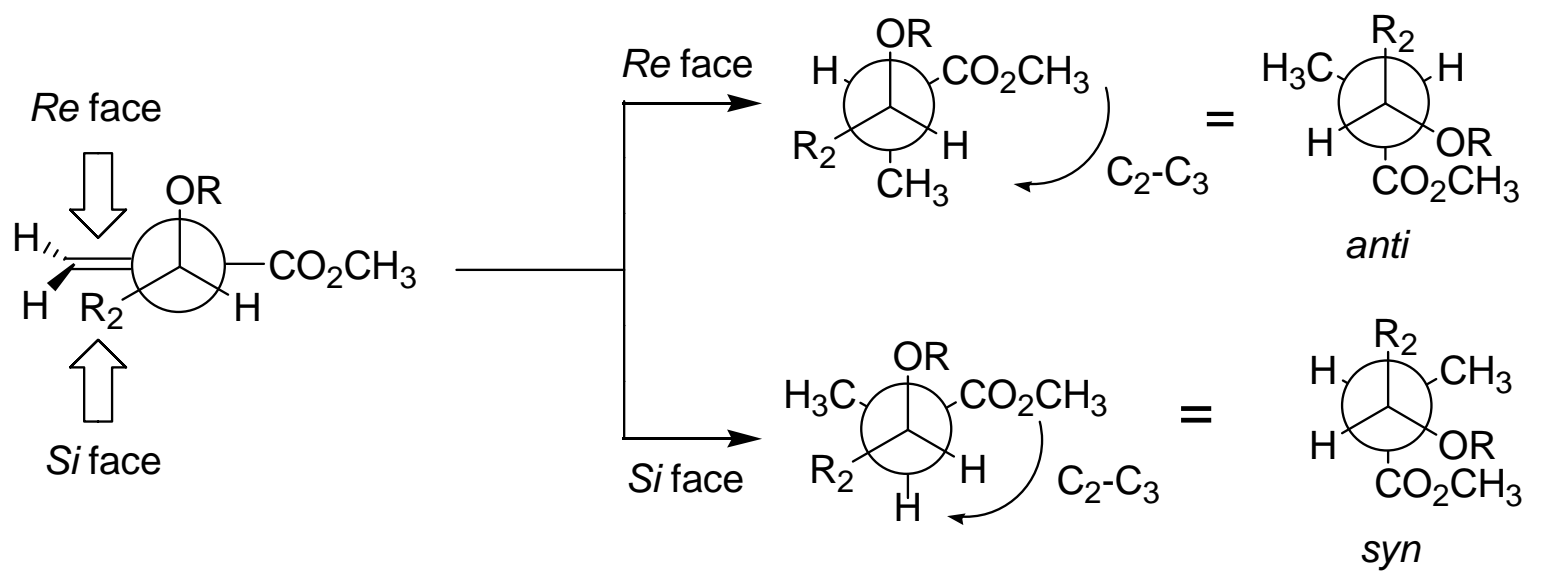

Scheme 5. Stereochemical rationalisation for the heterogeneous hydrogenation of BaylisHillman adducts.

For the cases we have studied, we observed the same stereochemical behaviour of silylated Baylis-Hillman adducts, which gave syn diastereoisomers as major products when submitted to the heterogeneous hydrogenation experimental conditions. For methyl ether derivatives the same behaviour, e.g. syn diastereoselectivity, has been observed, however the diastereoselectivity obtained in these cases was not as good as those described for the silylated ether derivatives. For acetylated derivatives, when diastereoselectivity was observed, it was very poor and the isomer ratios were inverted. In these cases the anti diastereoisomer were the major one.

\section{Conclusions}

This simple heterogeneous hydrogenation method of silylated Baylis-Hillman adducts could be considered as an alternative strategy to directly provide products which otherwise could only be obtained through an aldol condensation with propionate derivatives. However, the aldol condensation, despite its widespread utilisation, requires special experimental conditions, such as low temperatures and sophisticated bases. As it is possible to obtain enantiomerically pure Baylis-Hillman adducts (by resolution or through asymmetric base-catalysed reaction) their hydrogenation could also provide an easy and straighforward access to enantiomerically pure propionate derivatives.

Finally, this method should also be considered as an interesting, easier alternative to the homogeneous hydrogenation reaction of Weinreb amides obtained from Baylis-Hillman adducts. $^{15}$

\section{Experimental Section}


General Procedures. The ${ }^{1} \mathrm{H}$ and ${ }^{13} \mathrm{C}$ spectra were recorded on a Varian GEMINI BB-300 at 300 and $75.4 \mathrm{MHz}$, respectively, or on an Inova instrument at 500 and $125 \mathrm{MHz}$, respectively. The mass spectra were recorded using a HP model 5988A GC/MS with a High Resolution Autospec-Micromass/EBE. IR were obtained with a Nicolet model Impact 410. Melting points were measured in open capillary tubes using an Electrothermal model 9100, and are uncorrected. Yields were determined from GC analyses on a HP6890 equipment with a flame ionization detector, using a HP-5 capillary (crosslinked 5\% Phenyl methyl siloxanes, $28 \mathrm{~m}$ ) column. Manipulations and reactions were not performed under dry atmospheres or employing dry solvents, unless otherwise specified. Purification and separations by column chromatography were performed on silica gel, using normal or flash chromatography. TLC visualization was achieved by spraying with 5\% ethanolic phosphomolybdic acid and heating. All Baylis-Hillman reactions were sonicated in an ultrasonic cleaner UNIQUE model GA 1000 (1000 W, $25 \mathrm{kHz}$ ). Aromatic aldehydes and aliphatic aldehydes were purchased from Aldrich, Acros or Lancaster and were used without previous purification.

\section{General procedure for the protection of Baylis-Hillman adducts as trimethylsilyl ethers (Preparation of 15a, 16a, 17a, 19a)}

A mixture of $1 \mathrm{mmol}$ of the Baylis-Hillman adduct (1, 2, 3 and 5), 1.5 equivalents of trimethylsilylchloride (freshly distilled) and 1.2 equivalents of triethylamine (dried over $\mathrm{CaH}_{2}$ ) in $4 \mathrm{~mL}$ of dry tetrahydrofuran was stirred at room temperature, under nitrogen, for $6 \mathrm{~h}$ (the end of the reaction was confirmed by tlc). Then, the reaction medium was extracted with hexane ( $3 \times 20$ $\mathrm{mL}$ ). After washing with distilled water and drying over $\mathrm{Na}_{2} \mathrm{SO}_{4}$, the organic layer was evaporated under reduced pressure to furnish an oily residue which was purified by silica gel column chromatography.

Methyl 2-trimethylsilyloxy(phenyl)methylacrylate (15a). Eluent: hexane : ethyl acetate 90:10 $\mathrm{v} / \mathrm{v},\left(0.18 \mathrm{~g}\right.$ of a volatile and fluid liquid, 61\%). IR ( $v_{\max } /$ Film) 3087, 2954, 2856, $1722(\mathrm{C}=\mathrm{O})$, $1634(\mathrm{C}=\mathrm{C}) \mathrm{cm}^{-1}$; ${ }^{1} \mathrm{H}$ NMR $\left(300 \mathrm{MHz}, \mathrm{CDCl}_{3}\right) \delta 0.05$ (s, 9H, OSi $\left.\left(\underline{\mathrm{C}}_{3}\right)_{3}\right), 3.66$ (s, 3H, OC$\underline{\mathrm{H}}_{3}$ ester), 5.59 (s, $1 \mathrm{H}, \mathrm{CHOSiR}_{3}$ ), 6.20 (s, $1 \mathrm{H}$, double bond), 6.27 (s, $1 \mathrm{H}$, double bond), 7.22-7.33 (m, $5 \mathrm{H}$ aromatics); ${ }^{13} \mathrm{C}$ NMR $\left(125 \mathrm{MHz}, \mathrm{CDCl}_{3}\right) \delta 170.8,148.2,142.8,128,126.8,126.4,123.1$, 73.9, 52.2, 0.7; Anal. calcd for $\mathrm{C}_{14} \mathrm{H}_{20} \mathrm{O}_{3} \mathrm{Si}$ : C, $63.60 \%$; H, 7.62\%. Found C, 63.58\%; H, 7.60\%.

Methyl 2-[4-methoxyphenyl(trimethylsilyloxy)methyl]acrylate (16a). Eluent: hexane : ethyl acetate $95: 5 \mathrm{v} / \mathrm{v},\left(0.20 \mathrm{~g}\right.$ of a volatile and fluid liquid, 56\%). IR ( $v_{\max } /$ Film) 3087, 2954, 2856, $1722(\mathrm{C}=\mathrm{O}), 1632(\mathrm{C}=\mathrm{C}) \mathrm{cm}^{-1}$; ${ }^{1} \mathrm{H}$ NMR $\left(300 \mathrm{MHz}, \mathrm{CDCl}_{3}\right) \delta 0.05$ (s, 9H, OSi $\left.\left(\underline{\mathrm{C}}_{3}\right)_{3}\right), 3.67(\mathrm{~s}$, $3 \mathrm{H}, \mathrm{OCH}_{3}$ ester), 3.70 (s, $3 \mathrm{H}, \mathrm{OCH}_{3}$ aromatic ), 5.56 (s, $1 \mathrm{H}, \mathrm{CHOSiR}_{3}$ ), 6.0 (s, $1 \mathrm{H}$, double bond), 6.25 (s, $1 \mathrm{H}$, double bond), 6.83 (d, $J=8.5 \mathrm{~Hz}, 2 \mathrm{H}$ aromatics), 7.24 (d, $J=8.5 \mathrm{~Hz}, 2 \mathrm{H}$ aromatics); ${ }^{13} \mathrm{C}$ NMR $\left(125 \mathrm{MHz}, \mathrm{CDCl}_{3}\right) \delta 170.8,157.7,148.2,136.6,129.9,123.1,112.1,73.9,55.0,52.2$, 0.7; Anal. calcd for $\mathrm{C}_{15} \mathrm{H}_{22} \mathrm{O}_{4} \mathrm{Si}$ : C, 61.19\%; H, 7.53\%. Found C, 61.17\%; H, 7.52\%. 
Methyl 2-[4-chlorophenyl(trimethylsilyloxy)methyl]acrylate (17a). Eluent: hexane : ethyl acetate $90: 10 \mathrm{v} / \mathrm{v},\left(0.13 \mathrm{~g}\right.$ of a volatile and fluid liquid, 99\%). IR ( $v_{\max } /$ Film) 3087, 2954, 2856, $1720(\mathrm{C}=\mathrm{O}), 1630(\mathrm{C}=\mathrm{C}) \mathrm{cm}^{-1} ;{ }^{1} \mathrm{H}$ NMR ( 300MHz, $\left.\mathrm{CDCl}_{3}\right) \delta 0.02$ (s, 9H, OSi $\left.\left(\mathrm{C}_{3}\right)_{3}\right), 3.61$ (s, $3 \mathrm{H}, \mathrm{OC}_{3}$ ester), 5.12 (s large, $\mathrm{CHOSiR}_{3}$ ), 5.96 (s large, $1 \mathrm{H}$, double bond), 6.22 (s large, $1 \mathrm{H}$ ), 7.21-7.20 (m, 4H aromatics); ${ }^{13} \mathrm{C}$ NMR $\left(125 \mathrm{MHz}, \mathrm{CDCl}_{3}\right) \delta 170.8,148.2,141.8,132.5,129.0$, 126.7, 123.1, 73.9, 52.2, 0.8; Anal. calcd for $\mathrm{C}_{14} \mathrm{H}_{19} \mathrm{ClO}_{3} \mathrm{Si}$ : C, 56.27\%; H, 6.41\%. Found C, $56.27 \%$; H, 6.40\%.

Methyl 2-trimethylsilyloxy(4-nitrophenyl)methylacrylate (19a). Eluent: hexane : ethyl acetate 85:15 v/v, (0.25 g of a fluid liquid, 52\%). IR ( $v_{\max } /$ Film) 3087, 2954, 2856, 1725 (C=O), 1632 $(\mathrm{C}=\mathrm{C}) \mathrm{cm}^{-1}$; ${ }^{1} \mathrm{H}$ NMR $\left(300 \mathrm{MHz}, \mathrm{CDCl}_{3}\right) \delta 0.09$ (s, 9H, OSi $\left.\left(\underline{\mathrm{C}}_{3}\right)_{3}\right), 3.70$ (s, 3H, OC$\underline{\mathrm{H}}_{3}$ ester), 5.67 (s, 1H, $\mathrm{CHOSiR}_{3}$ ), 6.09 (s, $1 \mathrm{H}$, double bond), 6.34 (s, $1 \mathrm{H}$, double bond), 7.53 (d, $J=8.8 \mathrm{~Hz}$, $2 \mathrm{H}$ aromatics), 8.16 (d, $J=8.8 \mathrm{~Hz}, 2 \mathrm{H}$ aromatics); $\left.{ }^{13} \mathrm{C} \mathrm{NMR} \mathrm{(125MHz,} \mathrm{CDCl}_{3}\right) \delta 170.8,148.2$, 141.8, 132.5, 129.0, 126.7, 123.1, 73.9, 52.2, 0.8; Anal. calcd for $\mathrm{C}_{14} \mathrm{H}_{19} \mathrm{NO}_{5} \mathrm{Si}$ : C, 54.35\%; $\mathrm{H}$, 6.19\%. Found C, 54.33\%; H, 6.17\%.

\section{Preparation of methyl 2-benzo[d][1.3]dioxol-5-yl(trimethylsilyloxy)methylacrylate (18a).}

A mixture of $1 \mathrm{mmol}$ of Baylis-Hillman adducts (4), 1.5 equivalents of trimethylsilyl trifluoromethanesulfonate and 2 equivalents of anhydrous 2,6-lutidine in $0.5 \mathrm{~mL}$ of dichloromethane was stirred at room temperature, under a nitrogen atmosphere, for 30 minutes. After this time, the reaction medium was diluted with $60 \mathrm{~mL}$ of ethyl ether and the organic layer was successively washed with a $0.1 \mathrm{~mol} / \mathrm{L}$ solution of $\mathrm{HCl}$, a saturated solution of $\mathrm{NaHCO}_{3}$ and finally brine. Then, the organic layer was dried over anhydrous $\mathrm{Na}_{2} \mathrm{SO}_{4}$ and evaporated under reduced pressure to provide an oily residue, which was purified by silica gel column chromatography. Eluent: hexane : ethyl acetate $94: 6 \mathrm{v} / \mathrm{v},(0.18 \mathrm{~g}$ of a volatile and fluid liquid, 70\%). IR ( $v_{\max } /$ Film) 3087, 2954, 2856, $1722(\mathrm{C}=\mathrm{O}), 1634(\mathrm{C}=\mathrm{C}) \mathrm{cm}^{-1}$; ${ }^{1} \mathrm{H}$ NMR (300MHz,

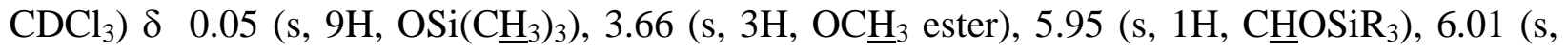
$2 \mathrm{H},-\mathrm{OC} \underline{\mathrm{H}}_{2} \mathrm{O}-$ ), 6.20 (s, $1 \mathrm{H}$, double bond), 6.27 (s, $1 \mathrm{H}$, double bond), 7.22-7.33 (m, $5 \mathrm{H}$ aromatics); ${ }^{13} \mathrm{C}$ NMR $\left(125 \mathrm{MHz}, \mathrm{CDCl}_{3}\right) \delta 170.8,148.2,142.8,146.8,145.6,136.8,120.4,109.8$, 107, 75.6, 52.2, 0.7; Anal. calcd for $\mathrm{C}_{15} \mathrm{H}_{20} \mathrm{O}_{5} \mathrm{Si}$ : C, 58.42\%; H, 6.54\%. Found C, 58.41\%; H, $6.52 \%$.

General procedure for the protection of Baylis-Hillman adducts as triethylsilyl, tertbutyldimethylsilyl and tert-butyldiphenysilyl ethers. (Preparation of 15b, 15d and 15e, 16b, 16d and 16e, 17b, 17d-e, 18b, 18d-e, 19b, 36-39)

A mixture of $1 \mathrm{mmol}$ of the Baylis-Hillman adduct, $1.3 \mathrm{mmol}$ of triethylsilyl chloride, tertbutyldimethylsilyl chloride or tert-butyldiphenylsilylchloride, $2.5 \mathrm{mmol}$ of imidazole and 0.3 $\mathrm{cm}^{3}$ of dry N,N-dimethylformamide was stirred at room temperature, under nitrogen, for 12-18 h (the end of reaction was confirmed by tlc). Then, the reaction medium was quenched with 10 $\mathrm{cm}^{3}$ of hexane. After washing with brine $\left(3 \times 5 \mathrm{~cm}^{3}\right)$, drying over $\mathrm{MgSO}_{4}$ and evaporation, the 
residue obtained was purified by silica gel column chromatography (eluent indicated for each adduct).

Methyl 2-triethylsilyloxy(phenyl)methylacrylate (15b). Eluent: hexane: ethyl acetate 92:8 v/v, (0.2 g of an oil, 70\%). IR ( $v_{\max } /$ Film) 3032, 2954, 2875, 1454, 1089, 1064, 1722 (C=O), 1630 $(\mathrm{C}=\mathrm{C}) \mathrm{cm}^{-1} ;{ }^{1} \mathrm{H}$ NMR $\left(500 \mathrm{MHz}, \mathrm{CDCl}_{3}\right) \delta$ 0.48-0.6 (m, 6H, OSiC플 $\left.\mathrm{CH}_{3}\right), 0.82-0.9(\mathrm{~m}, 9 \mathrm{H}$, $\mathrm{OSiCH}_{2} \mathrm{CH}_{3}$ ), 3.66 (s, 3H, $\mathrm{OCH}_{3}$ ester), 6.1 (s large, 1H, $\mathrm{CHOSiR}_{3}$ ), 6.25 (s, 1H, double bond), 6.26 (s, $1 \mathrm{H}$, double bond), 7.2-7.4 (m, 5H aromatics); ${ }^{13} \mathrm{C}$ NMR (125MHz, $\mathrm{CDCl}_{3}$ ) $\delta 171,147.4$, 141.3, 127.7, 126.8, 126.4, 122.6, 76, 52.2, 6.3, 6.1; Anal. calcd for $\mathrm{C}_{17} \mathrm{H}_{26} \mathrm{O}_{3} \mathrm{Si}$ : C, 66.62\%; $\mathrm{H}$, 8.55\%. Found C, 66.59\%; H, 8.53\%.

Methyl 2-tert-butyldimethylsilyloxy(phenyl)methylacrylate (15d). Eluent: hexane: ethyl acetate 90:10 v/v, (0.2 g of a viscous oil, 80\%). IR ( $v_{\max } /$ Film) 3087, 2954, 2856, 1722 (C=O), $1634(\mathrm{C}=\mathrm{C}), 1257,1088 \mathrm{~cm}^{-1}$; ${ }^{1} \mathrm{H}$ NMR $\left(300 \mathrm{MHz}, \mathrm{CDCl}_{3}\right) \delta-0.1$ (s, 6H, OSi $\left.\left(\mathrm{C}_{3}\right)_{2}\right), 0.9(\mathrm{~s}, 9 \mathrm{H}$,

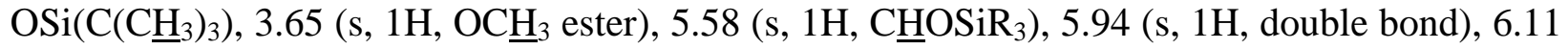
(s, $1 \mathrm{H}$, double bond), 7.10-7.33 (m, 5H aromatics); $\left.{ }^{13} \mathrm{C} \mathrm{NMR} \mathrm{(75.4MHz,} \mathrm{CDCl}_{3}\right) \delta$ 168.8, 138.3, 134.9, 130.9, 130.6, 127.1, 127.8, 125.8, 52.2, 45.6, 26.1, 20.8, -6.3; Anal. calcd for $\mathrm{C}_{17} \mathrm{H}_{26} \mathrm{O}_{3} \mathrm{Si}$ : C, 66.62\%; H, 8.55\%. Found C, 66.60\%; H, 8.54\%.

Methyl 2-tert-butyldiphenylsilyloxy(phenyl)methylacrylate (15e). Eluent: hexane: ethyl acetate 95:5 v/v, (0.19g of a viscous oil, 76\%). IR ( $v_{\max } /$ Film) 3087, 2954, 2856, 1724 (C=O), $\left.1632(\mathrm{C}=\mathrm{C}) \mathrm{cm}^{-1} ;{ }^{1} \mathrm{H} \mathrm{NMR}, 300 \mathrm{MHz}, \mathrm{CDCl}_{3}\right) \delta 1.0$ (s, 9H, OSi-t-Bu), 3.58 (s, 3H, OCH $\mathrm{OH}_{3}$ ester), 5.52 (s large, $1 \mathrm{H}, \mathrm{CHOSiR}_{3}$ ), 6.23 (s large, 2H, double bond), 7.15-7.62 (m, 15H aromatics, $\mathrm{Si}(\mathrm{Ph})_{2}+$ aromatics); ${ }^{13} \mathrm{C} \mathrm{NMR}\left(125 \mathrm{MHz}, \mathrm{CDCl}_{3}\right) \delta 170.8,148.2,136.1,133.1,129.9,127.8$, 127.7, 126.4, 126.8, 123.1, 80.9, 52.2, 22.6; Anal. calcd for $\mathrm{C}_{27} \mathrm{H}_{30} \mathrm{O}_{3} \mathrm{Si}$ : C, 75.31\%; H, 7.02\%. Found C, 75.29\%; H, 7.0\%.

Methyl 2-[triethylsilyloxy(4-methoxyphenyl)methyl]acrylate (16b). Eluent: hexane: ethyl acetate 95 : $5 \mathrm{v} / \mathrm{v}$, (0.22 g of a viscous oil, 66\%). IR ( $v_{\max } /$ Film) 2954, 2912, 2875, 2835, 1722 $(\mathrm{C}=\mathrm{O}), 1610$ (C=C), 1512, 1458, 1248, $1078 \mathrm{~cm}^{-1}$; ${ }^{1} \mathrm{H}$ NMR (500MHz, $\left.\mathrm{CDCl}_{3}\right) \delta$ 0.48-0.6 (m, 6H, OSiC $\underline{H}_{2} \mathrm{CH}_{3}$ ), 0.82-0.9 (m, 9H, OSiCH${ }_{2} \underline{\mathrm{CH}}_{3}$ ), 3.71 (s, 3H, OCH 3 ester), 3.79 (s, 3H, $\mathrm{OCH}_{3}$ aromatic), 5.56 (s, $1 \mathrm{H}, \mathrm{CHOSiR}_{3}$ ), 6.08 (s large, $1 \mathrm{H}$, double bond), 6.23 (s large, $1 \mathrm{H}$, double bond), 6.81 (d, $J=8.8 \mathrm{~Hz}, 2 \mathrm{H}$ aromatics), 7.26 (d, $J=8.8 \mathrm{~Hz}, 2 \mathrm{H}$ aromatics); ${ }^{13} \mathrm{C} \mathrm{NMR}$ $\left(125 \mathrm{MHz}, \mathrm{CDCl}_{3}\right) \delta 171,157.5,148.2,134.8,129.6,123.1,112.1,75.8,55,52.2,6.34,6.12$; Anal. calcd for $\mathrm{C}_{18} \mathrm{H}_{28} \mathrm{O}_{4} \mathrm{Si}$ : C, 64.25\%; H, 8.39\%. Found C, 64.23\%; H, 8.39\%.

Methyl 2-[tert-butyldimethylsilyloxy(4-methoxyphenyl)methyl]acrylate (16d). Eluent: hexane : ethyl acetate 95:5 v/v, (0.25 g of a viscous oil, 88\%). IR ( $v_{\max } /$ Film) 2954, 2875, 2835, $1720(\mathrm{C}=\mathrm{O}), 1632(\mathrm{C}=\mathrm{C}), \mathrm{cm}^{-1}$; ${ }^{1} \mathrm{H}$ NMR (300MHz, $\left.\mathrm{CDCl}_{3}\right) \delta-0.12$ (s, 3H, OSiC $\left.\underline{H}_{3}\right), 0.04$ (s, $3 \mathrm{H}, \mathrm{OSiC}_{3}$ ), 0.86 (s, 9H, $t$-BuSi), 3.66 (s, 3H, $\mathrm{OCH}_{3}$, ester), 3.78 (s, 3H, $\mathrm{OCH}_{3}$ aromatic), 5.54 (s large, 1H, Cي-HOSiR 3 ), 6.05 (s large, 1H, double bond), 6.21 (s large, 1H, double bond), 6.8 (d, $J=8.7 \mathrm{~Hz}, 2 \mathrm{H}$ aromatics), 7.23 (d, $J=8.7 \mathrm{~Hz}, 2 \mathrm{H}$ aromatics); ${ }^{13} \mathrm{C}$ NMR $\left(75.4 \mathrm{MHz}, \mathrm{CDCl}_{3}\right) 171$, 157.5, 149, 135, 129.6, 123, 112.1, 75.8, 55, 52.2, 25.8, 16.9, -4.5; Anal. calcd for $\mathrm{C}_{18} \mathrm{H}_{28} \mathrm{O}_{4} \mathrm{Si}$ : C, 64.25\%; H, 8.39\%. Found C, 64.25\%; H, 8.39\%. 
Methyl 2-[tert-butyldiphenylsilyloxy(4-methoxyphenyl)methyl]acrylate (16e). Eluent: hexane: ethyl acetate 95:5 v/v, (0.15 g of a viscous oil, 97\%). IR ( $v_{\max } /$ Film) $1722(\mathrm{C}=\mathrm{O}), 1629$ $(\mathrm{C}=\mathrm{C}), \mathrm{cm}^{-1}$; ${ }^{1} \mathrm{H}$ NMR $\left(300 \mathrm{MHz}, \mathrm{CDCl}_{3}\right) \delta 1.0$ (s, 9H), 3.5 (s, 3H, OCH $\mathrm{OCH}_{3}$ ester), $3.78\left(\mathrm{~s}, \mathrm{OCH}_{3}\right.$ aromatic), 5.54 (s large, 1H, HOSiR $_{3}$ ), 6.28 (s large, $2 \mathrm{H}$, double bond), 6.69 (d, $J=8.8 \mathrm{~Hz}, 2 \mathrm{H}$ aromatics), 7.0 (d, $J=8.8 \mathrm{~Hz}, 2 \mathrm{H}$ aromatics), 7.20-7.71 (m, $10 \mathrm{H}$ aromatics, $\left.-\mathrm{Si}(\mathrm{Ph})_{2}\right)$; ${ }^{13} \mathrm{C}$ NMR $\left(75.4 \mathrm{MHz}, \mathrm{CDCl}_{3}\right) \delta 170.9,157,147.9,136.1,133.1,129.9,129.6,127.8,112.1,80.9,55,52.2$, 22.6, 20; Anal. calcd for $\mathrm{C}_{28} \mathrm{H}_{32} \mathrm{O}_{4} \mathrm{Si}$ : C, 73.01\%; H, 7.00\%. Found C, 72.99\%; H, 6.98\%.

Methyl 2-[4-chlorophenyl(triethylsilyoxy)methyl]acrylate (17b). Eluent: hexane: ethyl acetate 95:5 v/v, (0.15 g of a fluid oil, 70\%). IR ( $v_{\max } /$ Film) $1723(\mathrm{C}=\mathrm{O}), 1632(\mathrm{C}=\mathrm{C}), \mathrm{cm}^{-1} ;{ }^{1} \mathrm{H}$ NMR $\left(300 \mathrm{MHz}, \mathrm{CDCl}_{3}\right) \delta$ 0.48-0.6 (m, 6H, OSiC $\left.\underline{\mathrm{H}}_{2} \mathrm{CH}_{3}\right), 0.82-0.9\left(\mathrm{~m}, 9 \mathrm{H}, \mathrm{OSiCH}_{2} \mathrm{CH}_{3}\right), 3.71(\mathrm{~s}, 3 \mathrm{H}$, $\mathrm{OCH}_{3}$ ester), 5.57 (s large, $1 \mathrm{H}, \mathrm{CHOSiR}_{3}$ ), 6.1 (s large, $1 \mathrm{H}$, double bond), 6.26 (s large, $1 \mathrm{H}$, double bond), 7.25-7.31 (m, 4H aromatics); $\left.{ }^{13} \mathrm{C} \mathrm{NMR} \mathrm{(75.4MHz,} \mathrm{CDCl}_{3}\right) \delta 171,148,140,128.7$, 126.7, 123.1, 75.8, 52.2, 6.34, 6.12; Anal. calcd for $\mathrm{C}_{17} \mathrm{H}_{25} \mathrm{ClO}_{3} \mathrm{Si}$ : C, 59.89\%; H, 7.39\%. Found C, 59.86\%; H, 7.37\%.

Methyl 2-[4-chlorophenyl(tert-butyldimethylsilyloxy)methyl]acrylate (17d). Eluent: hexane: ethyl acetate 95:05 v/v, (0.15 g of a viscous oil, 70\%). IR ( $v_{\max } /$ Film) $1717(\mathrm{C}=\mathrm{O}), 1634(\mathrm{C}=\mathrm{C}) \mathrm{cm}^{-1}$; ${ }^{1} \mathrm{H}$ NMR (300MHz, $\left.\mathrm{CDCl}_{3}\right) \delta 0.0$ (s, 6H, OSi $\left.\left(\underline{\mathrm{CH}}_{3}\right)_{2}\right), 0.9$ (s, 9H, OSi( $\left.\left.\mathrm{CH}_{3}\right)_{2} \underline{t-\mathrm{Bu}}\right), 3,67$ (s, 3H, $\mathrm{OCH}_{3}$ ester), 5.55 (s, $1 \mathrm{H}, \mathrm{CHOSiR}_{3}$ ), 6.13 (s, 1H, double bond), 6.32 (s, 1H, double bond), 7.20$8.24\left(\mathrm{~m}, 4 \mathrm{H}\right.$, aromatics); ${ }^{13} \mathrm{C} \mathrm{NMR}\left(75.4 \mathrm{MHz}, \mathrm{CDCl}_{3}\right) \delta 170.8,148.2,140.2,128.7,126.7$, 123.1, 75.8, 52.2, 25.8, 16.9, -4.56; Anal. calcd for $\mathrm{C}_{17} \mathrm{H}_{25} \mathrm{ClO}_{3} \mathrm{Si}$ : C, 59.89\%; H, 7.39\%. Found C, 59.89\%; H, 7.38\%.

Methyl 2-[4-chlorophenyl(tert-butyldiphenylsilyloxy)methyl]acrylate (17e). Eluent: hexane: ethyl acetate 95:05 v/v, (0.12 g of a viscous oil, 72\%). IR ( $v_{\max } /$ Film) $1722(\mathrm{C}=\mathrm{O}), 1630(\mathrm{C}=\mathrm{C}) \mathrm{cm}^{-1}$; ${ }^{1} \mathrm{H}$ NMR (300MHz, CDCl 3 ) $\delta 1.0$ (s, 9H, OSi-t-Bu), 3.54 (s, 3H, OC$\underline{H}_{3}$ ester), 5.52 (s large, 1H, $\mathrm{CHOSiR}_{3}$ ), 6.3 (s large, $2 \mathrm{H}, \mathrm{C}=\mathrm{CH}_{2}$ ), 7.0-7.12 (m, 4H aromatics), 7.21-7.8 (m, 10H aromatics, $\mathrm{Si}\left(\mathrm{C}_{6} \underline{\mathrm{H}}_{5}\right)_{2} ;{ }^{13} \mathrm{C} \mathrm{NMR}\left(75.4 \mathrm{MHz}, \mathrm{CDCl}_{3}\right) \delta 170.8,148.2,136.1,133.1,129.9,127.8,127.7,126.8$, 126.4, 80.9, 52.2, 22.6; Anal. calcd for $\mathrm{C}_{27} \mathrm{H}_{29} \mathrm{ClO}_{3} \mathrm{Si}$ : C, 69.73\%; H, 6.29\%. Found C, 69.71\%; H, 6.27\%.

Methyl 2-benzo[d][1,3]dioxol-5-yl(triethylsilyloxy)methylacrylate (18b). Eluent: hexane: ethyl acetate $90: 10 \mathrm{v} / \mathrm{v},\left(0.12 \mathrm{~g}\right.$ of a viscous oil, 88\%). IR ( $\mathrm{v}_{\max } /$ Film) 2959, 2877, 1722, 1630, 1489, 1442, 1238, $1078 \mathrm{~cm}^{-1}$; ${ }^{1} \mathrm{H}$ NMR $\left(300 \mathrm{MHz}, \mathrm{CDCl}_{3}\right) \delta$ 0.5-0.58 (m, 2H), 0.95-0.9 (m, 3H), 3.67 (s, 3H, $\mathrm{OCH}_{3}$ ester), 5.52 (s large, $1 \mathrm{H}, \mathrm{CHOSiR}_{3}$ ), 5.91 (s, $2 \mathrm{H},-\mathrm{OC}_{2} \underline{H}_{2} \mathrm{O}$ ), 6.0 (s large, $1 \mathrm{H}$, double bond), 6.23 (s large, $1 \mathrm{H}$, double bond), 6.70-6.84 (m, 3H aromatics); ${ }^{13} \mathrm{C} \mathrm{NMR}$ $\left(75.4 \mathrm{MHz}, \mathrm{CDCl}_{3}\right) \delta 171,148.2,146,145.6,135.3,123.1,120.2,109.8,107,52.2$, 6.34, 6.1; Anal. calcd for $\mathrm{C}_{18} \mathrm{H}_{26} \mathrm{O}_{5} \mathrm{Si}$ : C, 61.68\%; H, 7.48\%. Found C, 61.66\%; H, 7.48\%..

Methyl 2-benzo[d][1,3]dioxol-5-yl(tert-butyldimethylsilyloxy)methylacrylate (18d). Eluent: hexane: ethyl acetate 95:5 v/v, (0.35 g of a viscous oil, 77\%). IR ( $v_{\max } /$ Film) 1722 (C=O), 1630

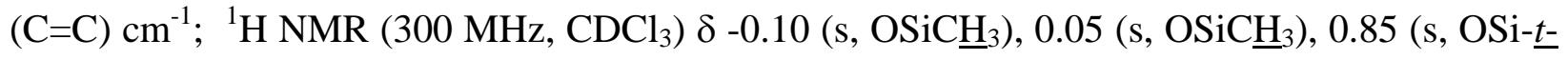
$\underline{\mathrm{Bu}}$ ), 3.7 (s, $\mathrm{OCH}_{3}$, ester), 5.5 (s, $1 \mathrm{H}, \mathrm{CHOSiR}_{3}$ ), 5.9 (s, $2 \mathrm{H},-\mathrm{OC}_{2} \mathrm{O}-$ ), 6.0 (s large, $1 \mathrm{H}$, double 
bond), 6.2 (s large, 1H, double bond), 6.7-6.9 (m, 3H aromatics); MS (70 eV, m/e, \%): 350 (M+, 0\%), 293 (100\%), 231 (10\%), 187 (13\%), 89 (23\%); HRMS (M+) Calcd. for $\mathrm{C}_{18} \mathrm{H}_{26} \mathrm{O}_{5} \mathrm{Si}$ 293,08429 - loss of $t$-butyl $\left(\mathrm{C}_{4} \mathrm{H}_{9}\right)$; Found: 350,154952 - 57,070425 = 293,084527.

Methyl 2-benzo[d][1,3]dioxol-5-yl(tert-butyldiphenylsilyloxy)methylacrylate (18e). Eluent: hexane: ethyl acetate 90:10 v/v, (0.2 g of a viscous oil, 93\%). IR ( $v_{\max } /$ Film) $1722(\mathrm{C}=\mathrm{O}), 1630$ $(\mathrm{C}=\mathrm{C}) \mathrm{cm}^{-1} ;{ }^{1} \mathrm{H}$ NMR (300 MHz, $\left.\mathrm{CDCl}_{3}\right) \delta 1.06$ (s, 9H, OSi-t-Bu), 3.53 (s, 3H, OCH 3 ester), 5.52 (s large, $1 \mathrm{H}, \mathrm{CHOSiR}_{3}$ ), 5.87 (m, 2H, - $\mathrm{OCH}_{2} \mathrm{O}-$ ), 6.27 (s, $1 \mathrm{H}$, double bond), 6.56 (s large, $1 \mathrm{H}$, double bond), 6.7 (s large, $1 \mathrm{H}$ aromatic), $7.23-7.72$ (m, 12H, aromatics); ${ }^{13} \mathrm{C} \mathrm{NMR}$ $\left(75.4 \mathrm{MHz}, \mathrm{CDCl}_{3}\right) \delta 169,148.2,146.8,145.6,136.2,133.1,129.9,127.8,123.1,120.2,109.6$, 107, 82.3, 52.2, 22.6, 20; Anal. calcd for $\mathrm{C}_{28} \mathrm{H}_{30} \mathrm{O}_{5} \mathrm{Si}$ : C, 70.86\%; H, 6.37\%. Found C, 70.86\%; H, $7.37 \%$.

Methyl 2-triethylsilyloxy(4-nitrophenyl)methylacrylate (19b). Eluent: hexane: ethyl acetate 90:10 v/v, (0.18 g of a fluid oil, 97\%). IR ( $v_{\max } /$ Film) $1722(\mathrm{C}=\mathrm{O}), 1630(\mathrm{C}=\mathrm{C}) \mathrm{cm}^{-1} ;{ }^{1} \mathrm{H}$ NMR $\left(300 \mathrm{MHz}, \mathrm{CDCl}_{3}\right) \delta$ 0.48-0.6 (m, 6H, OSiC $\left.\underline{\mathrm{H}}_{2} \mathrm{CH}_{3}\right), 0.82-0.9\left(\mathrm{~m}, 9 \mathrm{H}, \mathrm{OSiC}_{2} \mathrm{CH}_{3}\right), 3.75$ (s, $3 \mathrm{H}, \mathrm{OC}_{3}$ ester), 5.68 (s, $1 \mathrm{H}, \mathrm{CHOSiR}_{3}$ ), 6.19 (s, $1 \mathrm{H}$, double bond), 6.33 (s, $1 \mathrm{H}$, double bond), 7.56 (d, $J=8.8 \mathrm{~Hz}, 2 \mathrm{H}$ aromatics), 8.15 (d, $J=8.8 \mathrm{~Hz}, 2 \mathrm{H}$ aromatics); ${ }^{13} \mathrm{C} \mathrm{NMR}(75.4 \mathrm{MHz}$, $\left.\mathrm{CDCl}_{3}\right) \delta 169,150.3,148.2,145.1,130,123.1,120.8,75.8,51.5,6.3,6.1$; Anal. calcd for $\mathrm{C}_{17} \mathrm{H}_{25} \mathrm{NO}_{5} \mathrm{Si}$ : C, 58.09\%; H, 7.17\%. Found C, 58.07\%; H, 7.15\%.

Methyl 3-tert-butyldimethylsilyloxy-2-methylenebutanoate (36). Eluent: hexane-ethyl acetate, 90:10 v/v. (0.25 g of a viscous oil, 78\% yield). IR ( $v_{\max } /$ Film) $1717(\mathrm{C}=\mathrm{O}), 1630(\mathrm{C}=\mathrm{C}) \mathrm{cm}^{-1} ;{ }^{1} \mathrm{H}$

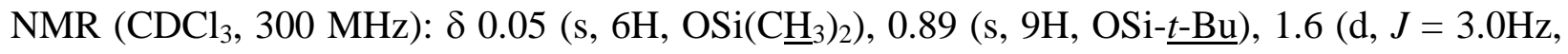
$3 \mathrm{H}$ ), 3.74 (s, $3 \mathrm{H}, \mathrm{OCH}_{3}$ ester), 4.4 (s large, $1 \mathrm{H}, \mathrm{CHOSiR}_{3}$ ), 6.2 (s large, $2 \mathrm{H}$, double bond); ${ }^{13} \mathrm{C}$ NMR (75MHz, $\left.\mathrm{CDCl}_{3}\right): \delta 171,148.9,121.3,69.9,52.2,25.8,21.5,-4.89$; Anal. calcd for $\mathrm{C}_{12} \mathrm{H}_{24} \mathrm{O}_{3} \mathrm{Si}$ : C, 58.97\%; H, 9.90\%. Found C, 58.95\%; H, 9.90\%.

Methyl 3-tert-butyldimethylsilyloxy-2-methylenepentanoate (37). Eluent: hexane-ethyl acetate, $90: 10 \mathrm{v} / \mathrm{v}$. (0.2 g of a viscous oil, 78\% yield). ${ }^{1} \mathrm{H} \mathrm{NMR}\left(\mathrm{CDCl}_{3}, 300 \mathrm{MHz}\right): \delta 0.05$ (s, 6H, Si $\left.\left(\underline{\mathrm{CH}}_{3}\right)_{2}\right), 0.89$ (s, 9H, OSi-t-Bu$), 0.91$ (t, $J=6.2 \mathrm{~Hz}, 3 \mathrm{H}, \mathrm{CH}_{3}$ terminal), $1.52-1.41$ (m,

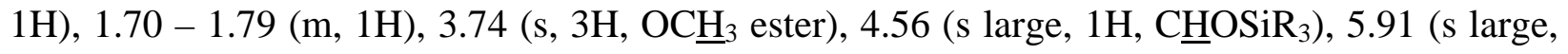
$1 \mathrm{H}$, double bond), 6.23 (s large, $1 \mathrm{H}$, double bond); ${ }^{13} \mathrm{C} \mathrm{NMR}\left(75.4 \mathrm{MHz}, \mathrm{CDCl}_{3}\right): \delta$ 166.8, 143.7, 124.6, 71.0, 51.6, 30.3, 25.8, 25.7, 25.6, 18.1, 9.1, -4.89,-4.83; Anal. calcd. for $\mathrm{C}_{13} \mathrm{H}_{36} \mathrm{O}_{3} \mathrm{Si} \mathrm{C}$ 58.20; H 13.43\%; Found C 58.17; H 13.41.

Methyl 3-tert-butyldimethylsilyloxy-2-methylenehexanoate (38). Eluent: hexane-ethyl acetate, 95:5 v/v. (0.2 g of a viscous oil, 77\% yield). ${ }^{1} \mathrm{H}$ NMR $\left(\mathrm{CDCl}_{3}, 300 \mathrm{MHz}\right) \delta-0.01$ (s, 3H), 0.06 (s, $3 \mathrm{H}), 0.92-0.83\left(\mathrm{~m}, 12 \mathrm{H}, \mathrm{CH}_{3}\right.$ terminal + OSi-t-Bu$), 1.69-1.45(\mathrm{~m}, 4 \mathrm{H}), 3.71\left(\mathrm{~s}, 3 \mathrm{H}, \mathrm{OCH}_{3}\right.$ ester), 4.57 (dd, $J=5.9$ and $4.8 \mathrm{~Hz}, 1 \mathrm{H}, \mathrm{CHOSiR}_{3}$ ), 5.86 (s large, $1 \mathrm{H}$, double bond), 6.22 (s large, $1 \mathrm{H}$, double bond); ${ }^{13} \mathrm{C} \mathrm{NMR}\left(\mathrm{CDCl}_{3}, 75.4 \mathrm{MHz}\right) \delta 166.6(\mathrm{C}=\mathrm{O}), 144.0,124.2,70.1,51.6$, 40.2, 25.9, 18.4, 18.2, 14.1, -4.5, -4.9; Anal. calcd for $\mathrm{C}_{14} \mathrm{H}_{28} \mathrm{O}_{3} \mathrm{Si}$ : C, 61.72\%; $\mathrm{H}, 10.36 \%$. Found C, $61.70 \% ; \mathrm{H}, 10.35 \%$. 
Methyl 3-tert-butyldimethylsilyloxy-2-methyleneheptanoate (39). Eluent: hexane-ethyl acetate, 95:5 v/v. (0.23 g of a viscous oil, 80\% yield). ${ }^{1} \mathrm{H} \mathrm{NMR}\left(\mathrm{CDCl}_{3}, 300 \mathrm{MHz}\right) \delta-0.01$ (s, $3 \mathrm{H}), 0.06$ (s, 3H), 0.91 (s, 12H, OSi-t-Bu $+\mathrm{CH}_{3}$ terminal), 1.64-1.24 (m, 6H), 3.76 (s, 3H, OC$\underline{H}_{3}$

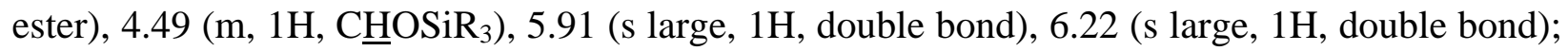
${ }^{13} \mathrm{C} \mathrm{NMR}\left(\mathrm{CDCl}_{3}, 300 \mathrm{MHz}\right) \delta 166.6(\mathrm{C}=\mathrm{O}), 144.0,124.2,70.2,51.6,37.6,27.2,25.9,25.7$, 22.6, 18.2, 17.1, 14.1, -4.5, -4.9; Anal. calcd for $\mathrm{C}_{15} \mathrm{H}_{30} \mathrm{O}_{3} \mathrm{Si}$ : C, 62.89\%; H, 10.55\%. Found C, $62.89 \% ; \mathrm{H}, 10.54 \%$.

\section{Alternative general procedure for the preparation of silylated Baylis-Hillman adducts (trialkyltriflate as silylating agent)}

A mixture of $1 \mathrm{mmol}$ of Baylis-Hillman adduct (1-5), 1.5 equivalents of trimethylsilyl trifluoromethanesulfonate, tri-isopropyl trifluoromethanesulfonate or tert-butyldimethylsilyl trifluoromethanesulfonate and 2 equivalents of anhydrous 2,6-lutidine in $0.5 \mathrm{~mL}$ of dichloromethane was stirred at room temperature, under a nitrogen atmosphere, for 30 minutes. After this time, the reaction medium was diluted with $60 \mathrm{~mL}$ of ethyl ether and the organic layer was successively washed with a $0.1 \mathrm{~mol} / \mathrm{L}$ solution of $\mathrm{HCl}$, a saturated solution of $\mathrm{NaHCO}_{3}$ and finally brine. Then, the organic layer was dried over anhydrous $\mathrm{Na}_{2} \mathrm{SO}_{4}$ and evaporated under reduced pressure to provide an oily residue, which was purified by silica gel column chromatography.

Methyl 2-tri-isopropylsilyloxy(phenyl)methylacrylate (15c). Eluent: hexane: ethyl acetate 95: $5 \mathrm{v} / \mathrm{v}\left(0.2 \mathrm{~g}\right.$ of a viscous colorless oil, 93\%). IR ( $v_{\max } /$ Film) $1719(\mathrm{C}=\mathrm{O}), 1630(\mathrm{C}=\mathrm{C}) \mathrm{cm}^{-1} ;{ }^{1} \mathrm{H}$ NMR $\left(300 \mathrm{MHz}, \mathrm{CDCl}_{3}\right) \delta$ 0.93-1.1 9 (m, 21H, OSi $\left.(\underline{i-P r})_{3}\right), 3.66$ (s, 3H, OC$\underline{H}_{3}$ ester), 5.70 (s, $1 \mathrm{H}, \mathrm{CHOSiR}_{3}$ ), 6.24 (s large, $2 \mathrm{H}$, double bond), 7.22-7.40 (m. 5H aromatics); ${ }^{13} \mathrm{C}$ NMR $\left(125 \mathrm{MHz}, \mathrm{CDCl}_{3}\right) \delta 170.8,148.2,139.7,127.4,126.8,126.4,123.1,77.5,52.2,15.5$, 9.9; Anal. calcd for $\mathrm{C}_{20} \mathrm{H}_{32} \mathrm{O}_{3} \mathrm{Si}$ : C, 68.92\%; H, 9.25\%. Found C, 68.90\%; H, 9.24\%.

Methyl 2-[4-methoxyphenyl(tri-isopropylsilyloxy)methyl]acrylate (16c). Eluent: hexane: ethyl acetate 92: $8 \mathrm{v} / \mathrm{v}$ (0.12 g of a viscous colorless oil, 83\%). IR ( $v_{\max } /$ Film) $1717(\mathrm{C}=\mathrm{O}), 1630$ $(\mathrm{C}=\mathrm{C}) \mathrm{cm}^{-1} ;{ }^{1} \mathrm{H}$ NMR $\left(300 \mathrm{MHz}, \mathrm{CDCl}_{3}\right) \delta$ 0.93-1.1 (m, 21H, OSi $\left.(\underline{i-P r})_{3}\right), 3.66\left(\mathrm{~s}, 3 \mathrm{H}, \mathrm{OC}_{3}\right.$ ester), 3.77 (s, $3 \mathrm{H}, \mathrm{OCH}_{3}$, aromatic), 5.65 (s, 1H, $\underline{\mathrm{HOSiR}}_{3}$ ), 6.21 (s large, $2 \mathrm{H}$, double bond), 6.79 (d, $J=8.8 \mathrm{~Hz}, 2 \mathrm{H}$ aromatics), 7.30 (d, $J=8.78 \mathrm{~Hz}, 2 \mathrm{H}$ aromatics); ${ }^{13} \mathrm{C} \mathrm{NMR} \mathrm{(75.4} \mathrm{MHz,}$ $\left.\mathrm{CDCl}_{3}\right) \delta 170,157.5,148.2,133.3,129.4,123.1,112.2,55,52.2$, 15.5, 9.9; Anal. calcd for $\mathrm{C}_{21} \mathrm{H}_{34} \mathrm{O}_{4} \mathrm{Si}$ : C, 66.62\%; H, 9.05\%. Found C, 66.62\%; H, 9.04\%.

Methyl 2-[4-chlorophenyl(tri-ispropylsilyloxy)methyl]acrylate (17c). Eluent: hexane: ethyl acetate $98: 2 \mathrm{v} / \mathrm{v}$ (0.17 $\mathrm{g}$ of a viscous colorless oil, 78\%). IR ( $v_{\max } /$ Film) $1721(\mathrm{C}=\mathrm{O}), 1632$ $(\mathrm{C}=\mathrm{C}) \mathrm{cm}^{-1} ;{ }^{1} \mathrm{H}$ NMR $\left(300 \mathrm{MHz}, \mathrm{CDCl}_{3}\right) \delta$ 0.93-1.1 (m, 21H, OSi $\left.(i-\mathrm{Pr})_{3}\right), 3.67\left(\mathrm{~s}, 3 \mathrm{H}, \mathrm{OCH}_{3}\right.$ ester), 5.67 (s, 1H, CㅂOSiR ), 6.23-6.26 (s large, 2H, double bond), 7.24 (d, $J=8.4 \mathrm{~Hz}, 2 \mathrm{H}$ aromatics), 7.3 (d, $J=8.4 \mathrm{~Hz}, 2 \mathrm{H}$ aromatics); ${ }^{13} \mathrm{C} \mathrm{NMR}\left(75.4 \mathrm{MHz}, \mathrm{CDCl}_{3}\right) \delta 166.7,144,141.7$, 132.9, 128.2, 128.1, 123.5, 71.9, 51.6, 17.9, 17.82, 12.1; Anal. calcd for $\mathrm{C}_{20} \mathrm{H}_{31} \mathrm{ClO}_{3} \mathrm{Si}$ : C, 62.72\%; H, 8.16\%. Found C, 62.70\%; H, 8.16\%. 
Methyl 2-benzo[d][1,3]dioxol-5-yl(tri-isopropylsilyloxy)methylacrylate (18c). Eluent: hexane: ethyl acetate $90: 10 \mathrm{v} / \mathrm{v}$ (0.19 g of a viscous colorless oil, 85\%); IR ( $v_{\max } /$ Film) 1720 $(\mathrm{C}=\mathrm{O}), 1632(\mathrm{C}=\mathrm{C}) \mathrm{cm}^{-1}$; ${ }^{1} \mathrm{H}$ NMR (300 MHz, $\left.\mathrm{CDCl}_{3}\right): \delta 0.86-1.11\left(\mathrm{~m}, 21 \mathrm{H}, \mathrm{OSi}(\underline{\underline{i}-\mathrm{Pr}})_{3}\right), 3.67$ (s, 3H, OC $\underline{H}_{3}$ ester), 5.60 (s large, $1 \mathrm{H}, \mathrm{C} \underline{\mathrm{HOTIPS}}$ ), 5.91 (s large, 2H, $\mathrm{OC}_{2} \mathrm{O}$ ), 6,21 (s large, 2H, $\mathrm{C}=\mathrm{C}_{2}$ ), 6.69 (s large, $1 \mathrm{H}$, aromatic), 6.78 (m, $2 \mathrm{H}$, aromatics); ${ }^{13} \mathrm{C} \mathrm{NMR}\left(75.4 \mathrm{MHz}, \mathrm{CDCl}_{3}\right) \delta$ 170, 148.2, 146.8, 146, 132, 123.1, 119.9, 109.3, 107, 79.1, 52.2, 15.5, 9.9; Anal. calcd for $\mathrm{C}_{21} \mathrm{H}_{32} \mathrm{O}_{5} \mathrm{Si}$ : C, 64.25\%; H, 8.22\%. Found C, 64.25\%; H, 8.21\%.

Methyl 2-tri-isopropylsilyloxy(4-nitrophenyl)methylacrylate (19c). Eluent: hexane: ethyl acetate $98: 2 \mathrm{v} / \mathrm{v}\left(0.22 \mathrm{~g}\right.$ of a viscous colorless oil, 94\%). IR ( $v_{\max } /$ Film) 1723 (C=O), 1634 $(\mathrm{C}=\mathrm{C}) \mathrm{cm}^{-1}$; ${ }^{1} \mathrm{H}$ NMR $\left(300 \mathrm{MHz}, \mathrm{CDCl}_{3}\right) \delta 1.0\left(\mathrm{~m}, 21 \mathrm{H}\right.$, OSi $\left.(\underline{i-P r})_{3}\right), 3.66$ (s, 3H, OC $\underline{H}_{3}$ ester), 5.65 (s large, $1 \mathrm{H}, \mathrm{CHOSiR}_{3}$ ), 6.26 (s large, $2 \mathrm{H}, \mathrm{C}_{2}-$ double bond), 7.5 (d, $J=8.0 \mathrm{~Hz}, 2 \mathrm{H}$ aromatics), 7.92 (d, $J=8.0 \mathrm{~Hz}, 2 \mathrm{H}$ aromatics); $\left.{ }^{13} \mathrm{C} \mathrm{NMR} \mathrm{(75.4MHz,} \mathrm{CDCl}_{3}\right) \delta 166.0,148.2$, 147.8, 145.1, 129.9, 120.8, 52.2, 15.5, 9.9; Anal. calcd for $\mathrm{C}_{20} \mathrm{H}_{31} \mathrm{NO}_{5} \mathrm{Si}: \mathrm{C}, 61.04 \%$; H, 7.94\%. Found C, 61.01\%; H, 7.93\%.

Methyl 2-tert-butyldimethylsilyloxy(4-nitrophenyl)methylacrylate (19d). Eluent: hexane: ethyl acetate 98: $2 \mathrm{v} / \mathrm{v}$ (0.13 g of a viscous colorless oil, 76\%). IR ( $v_{\max } /$ Film) $1717(\mathrm{C}=\mathrm{O}), 1634$

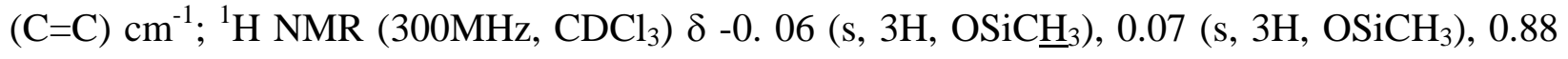
(s, 9H, Si-t-Bu), 3.69 (s, 3H, OC$\underline{H}_{3}$ ester), 5.68 (s, 1H, $\underline{\mathrm{HOSiR}}_{3}$ ), 6.17 (s, $1 \mathrm{H}$, double bond), 6.33 (s, $1 \mathrm{H}$, double bond), 7.56 (d, $J=8.5 \mathrm{~Hz}, 2 \mathrm{H}$ aromatics), 8.15 (d, $J=8.7 \mathrm{~Hz}, 2 \mathrm{H}$ aromatics); ${ }^{13} \mathrm{C} \mathrm{NMR}$ (75.4MHz, $\mathrm{CDCl}_{3}$ ) 165.8, 150.1, 147.2, 142.7, 127.7, 125.0, 123.4, 71.8, 51.8, 29.6, 25.6, 18.1, -4.9, -5.0; Anal. calcd for $\mathrm{C}_{17} \mathrm{H}_{25} \mathrm{NO}_{5} \mathrm{Si}$ : C, 58.09\%; H, 7.17\%. Found C, 58.08\%; H, $7.15 \%$.

\section{General procedure for acetylation of Baylis-Hillman adducts 20-24}

To a mixture of $1 \mathrm{mmol}$ of the Baylis-Hillman adducts (1-5) and 2.1 equivalents of triethylamine (dried over $\mathrm{CaH}_{2}$ and distilled) in $4 \mathrm{~mL}$ of dry dichloromethane were added, at $0^{\circ} \mathrm{C}, 1.5$ equivalents of acetyl chloride (freshly distilled). The reaction medium was stirred, at room temperature, under nitrogen, for 3-6 h (the end of the reaction was confirmed by tlc). Then, cold water $(3 \mathrm{~mL})$ was added and the reaction medium was diluted with dichloromethane $(15 \mathrm{~mL})$. After washing with distilled water, saturated solution of $\mathrm{NaHCO}_{3}(2 \times 15 \mathrm{~mL})$, brine (20 mL) and drying over $\mathrm{Na}_{2} \mathrm{SO}_{4}$, the organic layer was evaporated under reduced pressure to furnish an oily residue which was purified by silica gel column chromatography.

Methyl 2-methylcarbonyloxy(phenyl)methylacrylate (20). Eluent: hexane: ethyl acetate 80: 20 v/v (0.2 g of a tinged yellow oil, 82\%). IR ( $v_{\max } /$ Film) $1740(\mathrm{C}=\mathrm{O}), 1726(\mathrm{C}=\mathrm{O}), 1634(\mathrm{C}=\mathrm{C}) \mathrm{cm}^{-1} ;{ }^{1} \mathrm{H}$ NMR $\left(300 \mathrm{MHz}, \mathrm{CDCl}_{3}\right) \delta 2.31$ (s, 3H, $\mathrm{CH}_{3} \mathrm{CO}$ ), 3.91 (s, 3H, OC$\underline{H}_{3}$ ester), 5.89 (s, 1H, $\mathrm{CHOCOCH}_{3}$ ), $6.41\left(\mathrm{~s}, 1 \mathrm{H},=\mathrm{CH}_{2}\right), 6.70\left(\mathrm{~s}, 1 \mathrm{H},=\mathrm{CH}_{2}\right), 7.51-7.56(\mathrm{~m}, 5 \mathrm{H}$ aromatics$) ;{ }^{13} \mathrm{C}$ NMR $\left(75.4 \mathrm{MHz}, \mathrm{CDCl}_{3}\right) \delta 169.1,165.1,139.4,137.5,128.2,127.5,125.5,73,51.9,21.1$; MS (70eV, m/z): 191 ( $\mathrm{M}^{+}$- 43, 100\%), 175 (73\%), 159 (62\%), 132 (35\%), 115 (68\%), 105 (70\%), 77 (32\%); Anal. calcd for $\mathrm{C}_{13} \mathrm{H}_{14} \mathrm{O}_{4}$ : C, 66.66\%; H, 6.02\%. Found C, 66.64\%; H, 6.01\%. 
Methyl 2-[4-methoxyphenyl(methylcarbonyloxy)methyl]acrylate (21). Eluent: hexane: ethyl acetate $70: 30 \mathrm{v} / \mathrm{v}\left(0.08 \mathrm{~g}\right.$ of a colorless oil, 78\%). IR ( $v_{\max } /$ Film) $1733(\mathrm{C}=\mathrm{O}), 1719(\mathrm{C}=\mathrm{O})$, $1641(\mathrm{C}=\mathrm{C}) \mathrm{cm}^{-1} ;{ }^{1} \mathrm{H}$ NMR (300MHz, $\left.\mathrm{CDCl}_{3}\right) \delta 2.09$ (s, 3H, $\left.\underline{\mathrm{H}}_{3} \mathrm{CO}\right), 3.69$ (s, 3H, OC$\underline{H}_{3}$ ester), 3.79 (s, 3H, OC$\underline{H}_{3}$ aromatic), 5.87 (s, $\left.1 \mathrm{H}, \mathrm{CHOCOCH}_{3}\right), 6.37$ (s, $1 \mathrm{H}$, double bond), 6.62 (s, $1 \mathrm{H}$, double bond), 6.84 (d, $J=8.0 \mathrm{~Hz}, 2 \mathrm{H}$ aromatics), 7.31 (d, $J=8.0 \mathrm{~Hz}, 2 \mathrm{H}$ aromatics); ${ }^{13} \mathrm{C}$ NMR $\left(75,4 \mathrm{MHz}, \mathrm{CDCl}_{3}\right) \delta 169.2,163.2,159.4,139.5,129.6,129,125,113.7,72.8,55.2,52,21.2$; MS (70eV, m/z): $143\left(\mathrm{M}^{+}-\mathrm{COCH}_{3}, 77 \%\right), 127$ (100\%), 115 (85\%), 111 (80\%), 95 (60\%), 83 (48\%), 67 (90\%); Anal. calcd for $\mathrm{C}_{14} \mathrm{H}_{16} \mathrm{O}_{5}$ : C, 63.63\%; H, 6.10\%. Found C, 63.60\%; H, 6.09\%.

Methyl 2-[4-chlorophenyl(methylcarbonyloxy)methyl]acrylate (22). Eluent: hexane: ethyl acetate 70: $30 \mathrm{v} / \mathrm{v}\left(0.11 \mathrm{~g}\right.$ of a colorless oil, 80\%). IR ( $v_{\max } /$ Film) $1730(\mathrm{C}=\mathrm{O}), 1722(\mathrm{C}=\mathrm{O})$, $1634(\mathrm{C}=\mathrm{C}) \mathrm{cm}^{-1} ;{ }^{1} \mathrm{H}$ NMR (300MHz, $\left.\mathrm{CDCl}_{3}\right) \delta 2.0$ (s, 3H, $\left.\underline{\mathrm{C}}_{3} \mathrm{CO}\right), 3.74$ (s, 3H, OC$\underline{\mathrm{H}}_{3}$ ester), 6.25 (s large, $1 \mathrm{H}$ ), 6.44 (s large, $1 \mathrm{H}), 6.6$ (s, $1 \mathrm{H}), 7.20-7.33$ (m, 4H aromatics); ${ }^{13} \mathrm{C}$ NMR $\left(\mathrm{CDCl}_{3}, 75.4 \mathrm{MHz}\right) \delta 169,168.5,143.5,136.8,133.6,129.2,127.8,125.6,74,52.2$, 21; Anal. calcd for $\mathrm{C}_{13} \mathrm{H}_{13} \mathrm{ClO}_{4}$ : C, 58.11\%; H, 4.88\%. Found C, 58.08\%; H, 4.84\%.

Methyl 2-benzo[d][1,3]dioxol-5-yl(methylcarbonyloxy)methylacrylate (23). Eluent: hexane: ethyl acetate 85: $15 \mathrm{v} / \mathrm{v}\left(0.23 \mathrm{~g}\right.$ of a colorless oil, 89\%). IR ( $\left.v_{\max } / \mathrm{Film}\right) 1731(\mathrm{C}=\mathrm{O}), 1717(\mathrm{C}=\mathrm{O})$, $1630(\mathrm{C}=\mathrm{C}) \mathrm{cm}^{-1} ;{ }^{1} \mathrm{H}$ NMR $\left(300 \mathrm{MHz}, \mathrm{CDCl}_{3}\right) \delta 2.0$ (s, 3H, $\left.\underline{\mathrm{C}}_{3} \mathrm{CO}\right), 3.7$ (s, 3H, OC$\underline{\mathrm{H}}_{3}$ ester), 5.83 (s, $1 \mathrm{H}, \mathrm{CHOCOCH}_{3}$ ), 6.0 (s, $2 \mathrm{H},-\mathrm{OCH}_{2} \mathrm{O}-$ ), 6.42 (s large, $1 \mathrm{H}$, double bond), 6.44 (s large, $1 \mathrm{H}$, double bond), 7.0-7.12 (m, 3H aromatics); ${ }^{13} \mathrm{C} \mathrm{NMR}\left(\mathrm{CDCl}_{3}, 75.4 \mathrm{MHz}\right) \delta 170,168.5,147.9$, 146.7, 143.5, 132, 125.6, 120.7, 110, 108.1, 101.3, 76, 52.2, 21; Anal. calcd for $\mathrm{C}_{14} \mathrm{H}_{14} \mathrm{O}_{6}$ : C, 60.43\%; H, 5.07\%. Found C, 60.39\%; H, 4.99\%.

Methyl 2-methylcarbonyloxy(4-nitrophenyl)methylacrylate (24). Eluent: hexane: ethyl acetate 80: $20 \mathrm{v} / \mathrm{v}\left(0.15 \mathrm{~g}\right.$ of a viscous colorless oil, 76\%). IR ( $v_{\max } /$ Film) 1735 (C=O), 1719 $(\mathrm{C}=\mathrm{O}), 1632(\mathrm{C}=\mathrm{C}) \mathrm{cm}^{-1} ;{ }^{1} \mathrm{H}$ NMR $\left(300 \mathrm{MHz}, \mathrm{CDCl}_{3}\right) \delta 2.05$ (s, 3H, $\left.\underline{\mathrm{C}}_{3} \mathrm{CO}\right), 3.74$ (s, 3H, OC$\underline{\mathrm{H}}_{3}$ ester), 6.1 (s, $1 \mathrm{H}, \mathrm{CHOCOCH}_{3}$ ), 6.21 (s large, $1 \mathrm{H}$, double bond), 6.4 (s, 1H, double bond), 7.59 (d, $J=8.7 \mathrm{~Hz}, 2 \mathrm{H}$ aromatics), 7.82 (d, $J=8.7 \mathrm{~Hz}, 2 \mathrm{H}$ aromatics); ${ }^{13} \mathrm{C} \mathrm{NMR}\left(\mathrm{CDCl}_{3}, 75.4 \mathrm{MHz}\right) \delta$ 169.7, 168, 146.8, 146, 143.6, 130.7, 125.6, 121.9, 73.9, 52.2, 21.1; Anal. calcd for $\mathrm{C}_{13} \mathrm{H}_{13} \mathrm{NO}_{6}$ : C, 55.91\%; H, 4.69\%. Found C, 55.87\%; H, 4.65\%.

General procedure for methylation of Baylis-Hillman adducts 25-27. To a stirred solution of $1 \mathrm{mmol}$ of Baylis-Hillman adduct (1-3) in dichloromethane $(4 \mathrm{~mL})$ was added $\mathrm{Ag}_{2} \mathrm{O}(1.5-2$ mmol) and methyl iodide ( $3 \mathrm{mmol}$ ). The resulting suspension was then stirred at $40^{\circ} \mathrm{C}$ until complete consumption of the Baylis-Hillman adduct. The reaction was filtered and the solvent evaporated. The residue was further purified by column chromatography to provide the corresponding methyl ethers.

Methyl 2-methoxy(phenyl)methylacrylate (25). Eluent: hexane: ethyl acetate 70: $30 \mathrm{v} / \mathrm{v}(0.19 \mathrm{~g}$ of a viscous colorless oil, 86\%). IR ( $v_{\max } /$ Film) $1722(\mathrm{C}=\mathrm{O}), 1634(\mathrm{C}=\mathrm{C}) \mathrm{cm}^{-1}$; ${ }^{1} \mathrm{H}$ NMR $\left(300 \mathrm{MHz}, \mathrm{CDCl}_{3}\right) \delta 3.34$ (s, $3 \mathrm{H}, \mathrm{OCH}_{3}$ ether), 3.71 (s, $3 \mathrm{H}, \mathrm{OCH}_{3}$ ester), 5.15 (s, $1 \mathrm{H}, \mathrm{CHOCH}_{3}$ ), 5.94 (s, $1 \mathrm{H}$, double bond), 6.35 (s, $1 \mathrm{H}$, double bond), 7.29-7.36 (m, 5H aromatics); ${ }^{13} \mathrm{C}$ NMR 
$\left(\mathrm{CDCl}_{3}, 75.4 \mathrm{MHz}\right) \delta 165.4,140.9,139.1,127.1,123.9,80.5,56.2$, 51; Anal. calcd for $\mathrm{C}_{12} \mathrm{H}_{14} \mathrm{O}_{3}$ : C, 69.88\%; H, 6.84\%. Found C, 69.87\%; H, 6.84\%.

Methyl 2-methoxy(4-methoxyphenyl)methylacrylate (26). Eluent: hexane: ethyl acetate 70 : $30 \mathrm{v} / \mathrm{v}(0.198 \mathrm{~g}, 84 \%)$. IR ( $v_{\max } /$ Film) $1720(\mathrm{C}=\mathrm{O}), 1630(\mathrm{C}=\mathrm{C}), 1503 \mathrm{~cm}^{-1}$; $\left(300 \mathrm{MHz}, \mathrm{CDCl}_{3}\right)$

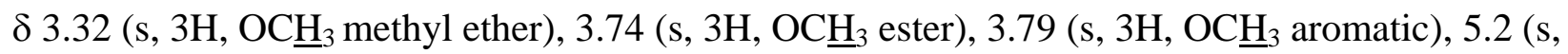
$1 \mathrm{H}, \mathrm{CHOCH}_{3}$ ), 6.15 (s, $1 \mathrm{H}$, double bond), 6.26 (s, $1 \mathrm{H}$, double bond), 6.73 (d, $J=8.8 \mathrm{~Hz}, 2 \mathrm{H}$ aromatics), 7.23 (d, $J=8.8 \mathrm{~Hz}, 2 \mathrm{H}$ aromatics); ${ }^{13} \mathrm{C} \mathrm{NMR}\left(125 \mathrm{MHz}, \mathrm{CDCl}_{3}\right) \delta 166.9,157.7,143$, 133.5, 130.2, 126.5, 113.5, 77.7, 56.6, 55, 52.2; Anal. calcd for $\mathrm{C}_{13} \mathrm{H}_{16} \mathrm{O}_{4}$ : C, 66.09\%; $\mathrm{H}, 6.83 \%$. Found C, 66.04\%; H, 6.80\%.

Methyl 2-[4-chlorophenyl(methoxy)methyl]acrylate (27). Eluent: hexane: ethyl acetate $70: 30$ v/v (0.187 g, 78\%). IR ( $v_{\max } /$ Film) $1720(\mathrm{C}=\mathrm{O}), 1633(\mathrm{C}=\mathrm{C}) \mathrm{cm}^{-1}$; (300MHz, CDCl $) \delta 3.3(\mathrm{~s}$, $3 \mathrm{H}, \mathrm{OC}_{3}$ methyl ether), 3.67 (s, $3 \mathrm{H}, \mathrm{OCH}_{3}$ ester), 4.83 (s, $1 \mathrm{H}, \mathrm{CHOCH}_{3}$ ), 6.15 (s, $1 \mathrm{H}$, double bond), 6.2 (s, 1H, double bond), 7.17-7.36 (m, 4H aromatics); $\left.{ }^{13} \mathrm{C} \mathrm{NMR} \mathrm{(125MHz,} \mathrm{CDCl}_{3}\right) \delta$ 166.7, 143, 138.9, 129.3, 126.5, 77.6, 56.6, 52.3; Anal. calcd for $\mathrm{C}_{12} \mathrm{H}_{3} \mathrm{ClO}_{3}$ : C, 59.88\%; $\mathrm{H}$, 5.44\%. Found C, 59.85\%; H, 5.42\%.

General procedure for the hydrogenation of Baylis-Hillman adducts. To a suspension of $5 \%$ Pd-C (10 mol\%) in ethyl acetate $\left(5 \mathrm{~cm}^{3}\right)$ was added, under nitrogen, a solution of the adduct $(1 \mathrm{mmol})$ in ethyl acetate $\left(5 \mathrm{~cm}^{3}\right)$. Then, the reaction atmosphere was changed to hydrogen and the reaction mixture was stirred at room temperature. After a period for reaction completion (30 min. to $2 \mathrm{~h}$ ), the suspension was filtered over a pad of Celite and the solvent was removed under reduced pressure (see chemical yields in Table 2). The residue was then treated with a solution of $5 \%$ HF in acetonitrile (1-3 $\mathrm{mL})$ or TBAF in THF (1-3 $\mathrm{mL}$ ) at room temperature for $2 \mathrm{~h}$. For the silylated adducts (15a-e/19a-d), after this time, the reaction mixture was diluted with ethyl acetate $(10-15 \mathrm{~mL})$ and water $(10-15 \mathrm{~mL})$. The phases were separated and the the organic layer was washed with distilled water $(10 \mathrm{~mL})$ and brine $(10 \mathrm{~mL})$, dried over $\mathrm{Na}_{2} \mathrm{SO}_{4}$ and evaporated to provide an oily residue sufficiently pure to be analysed without additional purification.

Methyl 3-hydroxy-2-methyl-3-phenylpropanoate (6a/b). Diastereoisomer syn (6a): ${ }^{1} \mathrm{H}$ NMR $\left(300 \mathrm{MHz}, \mathrm{CDCl}_{3}\right) \delta 1.15\left(\mathrm{~d}, J=7.69 \mathrm{~Hz}, 3 \mathrm{H}, \mathrm{CHCH}_{3}\right) 2.69-2.82(\mathrm{~m}, 1 \mathrm{H}$,

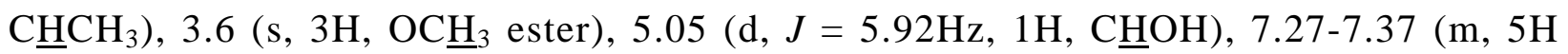
aromatics); $\left.{ }^{13} \mathrm{C} \mathrm{NMR} \mathrm{(300MHz,} \mathrm{CDCl}_{3}\right) \delta 173.6,136.7,128.1,127.3,126.8,73.4,51$, 38.5, 15; diastereoisomer anti (6b): ${ }^{1} \mathrm{H}$ NMR $\left(300 \mathrm{MHz}, \mathrm{CDCl}_{3}\right) \delta 0.90(\mathrm{~d}, J=6.9 \mathrm{~Hz}$, $3 \mathrm{H}, \mathrm{CHCH}_{3}$ ), 2.69-2.82 (m, 1H, $\left.\underline{\mathrm{HCH}}_{3}\right), 3.76$ (s, 3H, OC$\underline{H}_{3}$ ester), 4.73 (d, $J=8.1 \mathrm{~Hz}$, $1 \mathrm{H}, \mathrm{C} \underline{\mathrm{HOH}}), 7.27-7.37$ (m, 5H aromatics); $\left.{ }^{13} \mathrm{C} \mathrm{NMR} \mathrm{(300MHz,} \mathrm{CDCl}_{3}\right) \delta 175.6,137.6$, 128.1, 127.3, 126.8, 73.4, 51, 44.3, 16.4; Anal. calcd for $\mathrm{C}_{11} \mathrm{H}_{14} \mathrm{O}_{3}$ : C, 68.02\%; $\mathrm{H}$, 7.27\%. Found C, 68.00\%; H, 7.24\%. 
Methyl 3-hydroxy-3-(4-methoxyphenyl)-2-methylpropanoate (7a/b). Diastereoisomer syn (7a): ${ }^{1} \mathrm{H}$ NMR $\left(300 \mathrm{MHz}, \mathrm{CDCl}_{3}\right) \delta 1.14\left(\mathrm{~d}, J=6.9 \mathrm{~Hz}, 3 \mathrm{H}, \mathrm{CHCH}_{3}\right), 2.61-2.72(\mathrm{~m}, 1 \mathrm{H}$, $\mathrm{CHCH}_{3}$ ), 3.54 (s, $3 \mathrm{H}, \mathrm{OCH}_{3}$ ester), 3.79 (s, 3H, OCH ${ }_{3}$ ether), 4.91 (d, $J=6.2 \mathrm{~Hz}, 1 \mathrm{H}$, C $\underline{H O H}), 6.82$ (d, $J=8.79 \mathrm{~Hz}, 2 \mathrm{H}$ aromatics), 7.2 (d, $J=8.4 \mathrm{~Hz}, 2 \mathrm{H}$ aromatics); ${ }^{13} \mathrm{C}$ NMR $\left(300 \mathrm{MHz}, \mathrm{CDCl}_{3}\right) \delta 173,158.1,134.4,129.4,113.1,73.4,55,51,38.6,15$; diastereoisomer anti (7b). ${ }^{1} \mathrm{H} \operatorname{NMR}\left(300 \mathrm{MHz}, \mathrm{CDCl}_{3}\right) \delta 0.85(\mathrm{~d}, J=7.3 \mathrm{~Hz}, 3 \mathrm{H}$, $\left.\mathrm{CHCH}_{3}\right), 2.61-2.72\left(\mathrm{~m}, 1 \mathrm{H}, \mathrm{C}_{\mathrm{HCH}}\right), 3.54$ (s, $3 \mathrm{H}, \mathrm{OC}_{3}$ ester), 3.79 (s, $3 \mathrm{H}, \mathrm{OCH}_{3}$ ether), 4.64 (d, $J=9.8 \mathrm{~Hz}, 1 \mathrm{H}, \mathrm{CHOH}), 6.82(\mathrm{~d}, J=8.8 \mathrm{~Hz}, 2 \mathrm{H}$ aromatics), $7.2(\mathrm{~d}, J=$ 8.4Hz, $2 \mathrm{H}$ aromatics); ${ }^{13} \mathrm{C}$ NMR $\left(300 \mathrm{MHz}, \mathrm{CDCl}_{3}\right) \delta 175.6,158.1,134.4,129.4,113.2$, 73.4, 55, 51, 44.3, 16.4; Anal. calcd for $\mathrm{C}_{12} \mathrm{H}_{16} \mathrm{O}_{4}$ : C, 64.27\%; H, 5.19\%. Found C, $64.23 \%$; H, 5.16\%.

Methyl 3-(4-chlorophenyl)-3-hydroxy-2-methylpropanoate (8a/b). Diastereoisomer syn (8a): ${ }^{1} \mathrm{H}$ NMR $\left(300 \mathrm{MHz}, \mathrm{CDCl}_{3}\right) \delta 1.12$ (d, $\left.J=6.0 \mathrm{~Hz}, 3 \mathrm{H}\right), 2.55-2.74(\mathrm{~m}, 1 \mathrm{H}), 3.55$ $(\mathrm{s}, 3 \mathrm{H}), 4.95(\mathrm{~d}, J=6.1 \mathrm{~Hz}, 1 \mathrm{H}), 7.2-7.4\left(\mathrm{~m}, 4 \mathrm{H}\right.$ aromatics); ${ }^{13} \mathrm{C} \mathrm{NMR}(300 \mathrm{MHz}$, $\left.\mathrm{CDCl}_{3}\right) \delta 173.6,139.3,133.2,128.8,127.7,73.4,51,38.5,15$; diastereoisomer anti (8b): ${ }^{1} \mathrm{H}$ NMR $\left(300 \mathrm{MHz}, \mathrm{CDCl}_{3}\right) \delta 0.92(\mathrm{~d}, J=6.7 \mathrm{~Hz}, 3 \mathrm{H}), 2.55-2.74(\mathrm{~m}, 1 \mathrm{H}), 3.55$ (s, $3 \mathrm{H}), 4.67(\mathrm{~d}, J=9.2 \mathrm{~Hz}, 1 \mathrm{H}), 7.2-7.4(\mathrm{~m}, 4 \mathrm{H}$ aromatics $) ;{ }^{13} \mathrm{C}$ NMR $\left(300 \mathrm{MHz}, \mathrm{CDCl}_{3}\right) \delta$ 175.6, 139.3, 133.2, 128.8, 127.7, 73.4, 51, 44.3, 16.4; Anal. calcd for $\mathrm{C}_{11} \mathrm{H}_{13} \mathrm{ClO}_{3}$ : C, 57.78\%; H, 5.73\%. Found C, 57.75\%; H, 5.74\%.

Methyl 3-benzo[d][1,3]dioxol-5-yl-3-hydroxy-2-methylpropanoate (9a/b). Diastereoisomer syn (9a): ${ }^{1} \mathrm{H} \mathrm{NMR}\left(300 \mathrm{MHz}, \mathrm{CDCl}_{3}\right) \delta 1.14$ (d, $\left.J=6.95 \mathrm{~Hz}, 3 \mathrm{H}\right), 2.57-2.71(\mathrm{~m}, 1 \mathrm{H}$,

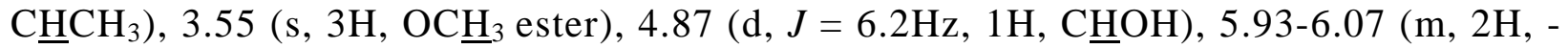
$\left.\mathrm{OC}_{2} \mathrm{O}-\right)$, 6.71-6.77 (m, $1 \mathrm{H}$ aromatic), 6.81-6.84 (m, $2 \mathrm{H}$ aromatics); ${ }^{13} \mathrm{C}$ NMR $\left(300 \mathrm{MHz}, \mathrm{CDCl}_{3}\right) \delta 173,147.8,146.3,135.9,120.7,110.3,108,101.3,74,50.9,38.5$,

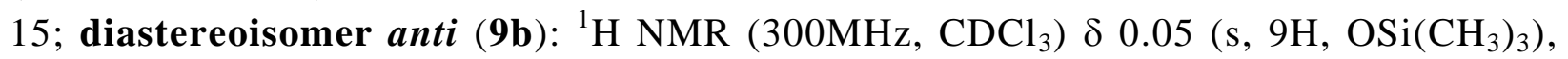
$1.7\left(\mathrm{~d}, J=6.9 \mathrm{~Hz}, 3 \mathrm{H}, \mathrm{CH}_{3}\right), 2.57-2.71\left(\mathrm{~m}, 1 \mathrm{H}, \mathrm{CHCH}_{3}\right), 3.71$ (s, 3H, $\mathrm{OCH}_{3}$ ester), 4.64 (d, $J=9.1 \mathrm{~Hz}, 1 \mathrm{H}, \mathrm{C} \underline{\mathrm{HOH}}), 5.93-6.0$ (m, 2H, $\left.-\mathrm{OC}_{2} \mathrm{O}-\right), 6.71-6.77$ (m, $1 \mathrm{H}$ aromatic), 6.81-6.84 (m, $2 \mathrm{H}$ aromatics); $\left.{ }^{13} \mathrm{C} \mathrm{NMR} \mathrm{(300MHz,} \mathrm{CDCl}_{3}\right) \delta 175.5,147.8,146.3,135.5$, 120.7, 110.3, 108, 101.3, 74, 51, 44.3, 16.3; Anal. calcd for $\mathrm{C}_{12} \mathrm{H}_{14} \mathrm{O}_{5}$ : C, 60.50\%; $\mathrm{H}$, 5.92\%. Found C, 60.49\%; H, 5.90\%.

Methyl 3-(4-nitrophenyl)-3-hydroxy-2-methylpropanoate (10a/b). Diastereoisomer syn (10a): ${ }^{1} \mathrm{H}$ NMR $\left(300 \mathrm{MHz}, \mathrm{CDCl}_{3}\right) \delta 1.15(\mathrm{~d}, J=6.61 \mathrm{~Hz}, 3 \mathrm{H}), 2.53-2.72(\mathrm{~m}, 1 \mathrm{H})$, $3.55(\mathrm{~s}, 3 \mathrm{H}), 4.72(\mathrm{~d}, J=6.6 \mathrm{~Hz}, 1 \mathrm{H}), 6.62(\mathrm{~d}, J=8.0 \mathrm{~Hz}, 2 \mathrm{H}$ aromatics$), 7.07(\mathrm{~d}, J=$ 8.4Hz, $2 \mathrm{H}$ aromatics); ${ }^{13} \mathrm{C}$ NMR $\left(300 \mathrm{MHz}, \mathrm{CDCl}_{3}\right) \delta 173.6,149.5,145.7,130.1,121.8$, 73.4, 51, 38.5, 15; diastereoisomer anti (10b): ${ }^{1} \mathrm{H}$ NMR $\left(300 \mathrm{MHz}, \mathrm{CDCl}_{3}\right) \delta 0.86$ (d, $J$

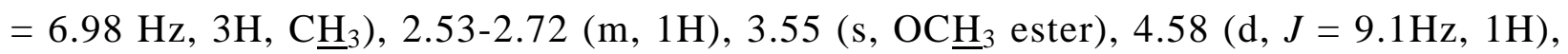
$6.62(\mathrm{~d}, J=8.0 \mathrm{~Hz}, 2 \mathrm{H}$ aromatics $) 7.07(\mathrm{~d}, J=8.0 \mathrm{~Hz}, 2 \mathrm{H}$ aromatics $) ;{ }^{13} \mathrm{C} \mathrm{NMR}$ $\left(300 \mathrm{MHz}, \mathrm{CDCl}_{3}\right) \delta 176,149.5,145.7,130.2,121.8,73,49.9,44.3$, 16.4; Anal. calcd for $\mathrm{C}_{11} \mathrm{H}_{13} \mathrm{NO}_{5}$ : C, 55.23\%; H, 5.48\%. Found C, 55.20\%; H, 5.47\%. 
Methyl 2-methyl-3-methylcarbonyloxy-3-phenylpropanoate (28a/b). Diastereoisomer syn (28a): ${ }^{1} \mathrm{H}$ NMR $\left(300 \mathrm{MHz}, \mathrm{CDCl}_{3}\right) \delta 1.16(\mathrm{~d}, J=6.5 \mathrm{~Hz}, 3 \mathrm{H}), 2.0$ (s, 3H, COCH 3 ), 2.86-2.90 (m, $1 \mathrm{H}), 3.65(\mathrm{~s}, 3 \mathrm{H}), 5.71(\mathrm{~d}, J=7.0 \mathrm{~Hz}, 1 \mathrm{H}), 7.21-7.30(\mathrm{~m}, 5 \mathrm{H}$ aromatics $) ;{ }^{13} \mathrm{C}$ NMR $\left(300 \mathrm{MHz}, \mathrm{CDCl}_{3}\right) \delta 174.3,171.3,136.5,128.4,127.2 .126 .7,80.5,51,36.4,21.4,16.8$; diastereoisomer anti (28b): ${ }^{1} \mathrm{H} \mathrm{NMR}\left(300 \mathrm{MHz}, \mathrm{CDCl}_{3}\right) \delta 1.05(\mathrm{~d}, J=6.5 \mathrm{~Hz}, 3 \mathrm{H}), 2.05$ (s, 3H), 2.87-2.93 (m, 1H), $3.62(\mathrm{~s}, 3 \mathrm{H}), 5.92(\mathrm{~d}, J=4.6 \mathrm{~Hz}, 1 \mathrm{H}), 7.26$ (s large, $5 \mathrm{H}$ aromatics) $;{ }^{13} \mathrm{C}$ NMR $\left(300 \mathrm{MHz}, \mathrm{CDCl}_{3}\right) \delta 176.3,171.3,136.5,128.4,127.2,126.9$, 80.5, 51, 42.2, 21.3, 18.2; Anal. calcd for $\mathrm{C}_{13} \mathrm{H}_{16} \mathrm{O}_{4}$ : C, 66.09\%; H, 6.83\%. Found C, 66.05\%; H, 6.82\%.

Methyl 3-(4-methoxyphenyl)-2-methyl-3-methylcarbonyloxypropanoate (29a/b). Diastereoisomer syn (29a): ${ }^{1} \mathrm{H}$ NMR (300MHz, $\left.\mathrm{CDCl}_{3}\right) \delta 1.14$ (d, J=6.3Hz, 3H), 2.05 (s, 3H, COC $\left.\underline{H}_{3}\right), 2.81-2.93(\mathrm{~m}, 1 \mathrm{H}), 3.59(\mathrm{~s}, 3 \mathrm{H}), 3.73(\mathrm{~s}, 3 \mathrm{H}), 5.71(\mathrm{~d}, J=7.0 \mathrm{~Hz}, 1 \mathrm{H})$, $6.79\left(\mathrm{~d}, J=8.8 \mathrm{~Hz}, 2 \mathrm{H}\right.$ aromatics), $7.05\left(\mathrm{~d}, J=8.8 \mathrm{~Hz}, 2 \mathrm{H}\right.$ aromatics); ${ }^{13} \mathrm{C} \mathrm{NMR}$ $\left(75.4 \mathrm{MHz}, \mathrm{CDCl}_{3}\right) \delta 174.3,171.3,158.2,134,129.6,113.1,80.5,55,51,36.4,21.4,16.8$; diastereoisomer anti (29b): ${ }^{1} \mathrm{H} \mathrm{NMR}\left(300 \mathrm{MHz}, \mathrm{CDCl}_{3}\right) \delta 1.14(\mathrm{~d}, J=6.5 \mathrm{~Hz}, 3 \mathrm{H}), 2.0(\mathrm{~s}, 3 \mathrm{H})$, 3.62 (s, 3H), 3.75 (s, 3H), 5.92 (d, J = 5.3Hz, 1H), 6.79 (d, $J=8.8 \mathrm{~Hz}, 2 \mathrm{H}$ aromatics), 7.05 (d, $J=$ $8.8 \mathrm{~Hz}, 2 \mathrm{H}$ aromatics); ${ }^{13} \mathrm{C}$ NMR $\left(75.4 \mathrm{MHz}, \mathrm{CDCl}_{3}\right) \delta 176.2,171.3,158.2,134,129.6,113.1$, 80.5, 55, 51, 42.2, 21.4, 18.2; Anal. calcd for $\mathrm{C}_{14} \mathrm{H}_{18} \mathrm{O}_{4}$ : C, 63.15\%; H, 6.81\%. Found C, 63.13\%; H, 6.78\%.

Methyl 3-(4-chlorophenyl)-2-methyl-3-methylcarbonyloxypropanoate (30a/b). Diastereoisomer syn (30a): ${ }^{1} \mathrm{H}$ NMR (300MHz, $\left.\mathrm{CDCl}_{3}\right) \delta 1.15$ (d, $\left.J=6.5 \mathrm{~Hz}, 3 \mathrm{H}\right), 2,05$ (s, 3H), 2.86-2.93 (m, 1H), 3.65 (s, 3H), 5.71 (d, $J=7.0 \mathrm{~Hz}, 1 \mathrm{H}), 7.28-7.31$ (m, $4 \mathrm{H}$ aromatics); ${ }^{13} \mathrm{C}$ NMR $\left(75.4 \mathrm{MHz}, \mathrm{CDCl}_{3}\right)$ $\delta$ 174.3, 171.2, 137.2, 133.3, 128.9, 127.6, 80.5, 51, 36.4, 21.3, 16.7; diastereoisomer anti (30b): ${ }^{1} \mathrm{H}$ NMR (300MHz, $\left.\mathrm{CDCl}_{3}\right) \delta 1.12$ (d, J = 6.4Hz, 3H), 2.0 (s, 3H), 2.84-2.90 (m, 1H), $3.63(\mathrm{~s}, 3 \mathrm{H}), 5.92(\mathrm{~d}, J=5.2 \mathrm{~Hz}, 1 \mathrm{H}), 7.28-7.31$ (m, 4H aromatics); ${ }^{13} \mathrm{C} \mathrm{NMR}(75.4 \mathrm{MHz}$, $\left.\mathrm{CDCl}_{3}\right) \delta 176.2,171.3,137.2,133.3,128.9,127.7,80.5,51,42.2,21.4,17.8$; Anal. calcd for $\mathrm{C}_{13} \mathrm{H}_{15} \mathrm{ClO}_{4}$ : C, 57.68\%; H, 5.59\%. Found C, 57.65\%; H, 5.57\%.

Methyl 3-benzo[d][1,3]dioxol-5-yl-2-methyl-3-methylcarbonyloxypropanoate (31a/b). Diastereoisomer syn (31a): ${ }^{1} \mathrm{H}$ NMR $\left(300 \mathrm{MHz}, \mathrm{CDCl}_{3}\right) \delta 1.13(\mathrm{~d}, J=6.5 \mathrm{~Hz}, 3 \mathrm{H}), 2.05(\mathrm{~s}, 3 \mathrm{H})$, 2.80-2.93 (m, 1H), 3.68 (s, 3H), 5.84 (d, $J=7.0 \mathrm{~Hz}, 1 \mathrm{H}), 6.05$ (s, 2H), 6.68-6.79 (m, 3H aromatics); ${ }^{13} \mathrm{C}$ NMR $\left(75.4 \mathrm{MHz}, \mathrm{CDCl}_{3}\right) \delta 174.3,171.3,147.7,146.5,132.7,119.7,110.5$, 107.9, 101.3, 81.8, 50.9, 36.5, 21.3, 16.8; diastereoisomer anti (31b): ${ }^{1} \mathrm{H} \mathrm{NMR} \mathrm{(300MHz,}$ $\left.\mathrm{CDCl}_{3}\right) \delta 1.16(\mathrm{~d}, J=6.5 \mathrm{~Hz}, 3 \mathrm{H}), 2.05$ (s, 3H), 2.87-2.93 (m. 1H), 3.64 (s, 3H), 6.05 (s, 2H), $6.08(\mathrm{~d}, J=5.3 \mathrm{~Hz}, 1 \mathrm{H}), 6.68-6.79\left(\mathrm{~m}, 3 \mathrm{H}\right.$ aromatics); ${ }^{13} \mathrm{C} \mathrm{NMR}\left(75.4 \mathrm{MHz}, \mathrm{CDCl}_{3}\right) \delta 176.3$, 171.3, 147.7, 146.4, 132.7, 119.7, 119.7, 110.5, 107.9, 101.3, 81.8, 51, 42.2, 21.4, 18; Anal. calcd for $\mathrm{C}_{14} \mathrm{H}_{16} \mathrm{O}_{6}$ : C, 59.99\%; H, 5.75\%. Found C, 59.99\%; H, 5.83\%.

Methyl 2-methyl-3-methylcarbonyloxy-3-(4-nitrophenyl)propanoate (32a/b). Diastereoisomer syn (32a): ${ }^{1} \mathrm{H}$ NMR (300MHz, $\left.\mathrm{CDCl}_{3}\right) \delta 1.16$ (d, $\left.J=6.4 \mathrm{~Hz}, 3 \mathrm{H}\right), 2.05$ (s, 3H), 2.83-2.91 (m, 1H), 3.60 (s, 3H), 5.71 (d, $J=7.0 \mathrm{~Hz}, 1 \mathrm{H}), 7.0$ (d, $J=8.9 \mathrm{~Hz}, 2 \mathrm{H}$ aromatics), 7.81 (d, $J=8.9 \mathrm{~Hz}, 2 \mathrm{H}$ 
aromatics); ${ }^{13} \mathrm{C}$ NMR (75.4MHz, $\left.\mathrm{CDCl}_{3}\right) \delta 174.3,171.3,146.4,145.9,130.3,121.7,80.5,51$, 36.4, 21.3, 16.7; diastereoisomer anti (32b): ${ }^{1} \mathrm{H} \mathrm{NMR}\left(300 \mathrm{MHz}, \mathrm{CDCl}_{3}\right) \delta 1.17$ (d, $J=6.0 \mathrm{~Hz}$, $3 \mathrm{H}), 2.0$ (s, 3H), 2.86-2.93 (m, 1H), 3.67 (s, 3H), 5.92 (d, $J=5.3 \mathrm{~Hz}, 1 \mathrm{H}), 7.0$ (d, $J=8.9 \mathrm{~Hz}, 2 \mathrm{H}$ aromatics), 7.81 (d, $J=8.9 \mathrm{~Hz}, 2 \mathrm{H}$ aromatics); ${ }^{13} \mathrm{C} \mathrm{NMR}\left(75.4 \mathrm{MHz}, \mathrm{CDCl}_{3}\right) \delta 176.3,171.3$, 146.4, 145.9, 130.3, 121.7, 80.5, 51, 42.2, 21.3, 18.2. Anal. calcd for $\mathrm{C}_{13} \mathrm{H}_{15} \mathrm{NO}_{6}$ : C, 55.51\%; $\mathrm{H}$, 5.38\%. Found C, 55.51\%; H, 5.38\%.

Methyl-3-methoxy-2-methyl-3-phenylpropanoate (33a/b). Diastereoisomer syn (33a): ${ }^{1} \mathrm{H}$ NMR (500MHz, $\left.\mathrm{CDCl}_{3}\right) \delta 1.23(\mathrm{~d}, J=6.95 \mathrm{~Hz}, 3 \mathrm{H}), 2.78(\mathrm{dq}, J=10$ and $6.95 \mathrm{~Hz}, 1 \mathrm{H}), 3.24$ (s, 3H), 3.55 (s, 3H), 4.44 (d, $J=6.95 \mathrm{~Hz}, 1 \mathrm{H}), 7.29-7.36$ (m, $5 \mathrm{H}$ aromatics); ${ }^{13} \mathrm{C}$ NMR $(125 \mathrm{MHz}$, $\left.\mathrm{CDCl}_{3}\right) \delta 175.8,138.9,128.5,128.3,127.7,86,56.8,51.8,47,14$; diastereoisomer anti (33b) ${ }^{1} \mathrm{H}$ NMR (500MHz, $\mathrm{CDCl}_{3}$ ) $\delta 0.88$ (d, $\left.J=6.5 \mathrm{~Hz}, 3 \mathrm{H}\right), 2.75$ (m, 1H), 3.15 (s, 3H), 3.76 (s, 3H), 4.25 (d, J= 10Hz, 1H), 7.29-7.36 (m, 5H aromatics); ${ }^{13} \mathrm{C}$ NMR (125MHz, $\left.\mathrm{CDCl}_{3}\right) \delta$ 174.4, 139.7, 128.2, 127.8, 127.0, 84.3, 57.1, 51.4, 47.4, 12.3; Anal. calcd for $\mathrm{C}_{14} \mathrm{H}_{14} \mathrm{O}_{6}$ : C, 60.43\%; $\mathrm{H}, 5.07 \%$. Found C, 60.39\%; H, 4.99\%.

Methyl-3-methoxy-3-(4-methoxyphenyl)-2-methylpropanoate (34a/b). Diastereoisomer syn (34a): ${ }^{1} \mathrm{H}$ NMR $\left(500 \mathrm{MHz}, \mathrm{CDCl}_{3}\right) \delta 1.12$ (d, $\left.J=6.5 \mathrm{~Hz}, 3 \mathrm{H}\right), 2.72-2.79(\mathrm{~m}, 1 \mathrm{H}), 3.35$ (s, 3H), $3.63(\mathrm{~s}, 3 \mathrm{H}), 3.72(\mathrm{~s}, 3 \mathrm{H}), 4.41(\mathrm{~d}, J=6.0 \mathrm{~Hz}, 1 \mathrm{H}), 6.82(\mathrm{~m}, 2 \mathrm{H}$ aromatics), $7.0(\mathrm{~m}, 2 \mathrm{H}$ aromatics); ${ }^{13} \mathrm{C}$ NMR $\left(125 \mathrm{MHz}, \mathrm{CDCl}_{3}\right) \delta 172.8,157.5,134.8,129.6,113.3,82.3,56,55,51$, 36.9, 15; diastereoisomer anti (34b) ${ }^{1} \mathrm{H}$ NMR $\left(500 \mathrm{MHz}, \mathrm{CDCl}_{3}\right) \delta 1.12(\mathrm{~d}, J=6.5 \mathrm{~Hz}$, $3 \mathrm{H}), 2.72-2.80(\mathrm{~m}, 1 \mathrm{H}), 3.40(\mathrm{~s}, 3 \mathrm{H}), 3.65(\mathrm{~s}, 3 \mathrm{H}), 3.75(\mathrm{~s}, 3 \mathrm{H}), 4.42$ (d, $J=6.0 \mathrm{~Hz}$, $1 \mathrm{H}), 6.82(\mathrm{~m}, 2 \mathrm{H}$ aromatics $), 7.0\left(\mathrm{~m}, 2 \mathrm{H}\right.$ aromatics); ${ }^{13} \mathrm{C} \mathrm{NMR}\left(125 \mathrm{MHz}, \mathrm{CDCl}_{3}\right) \delta$ 174.8, 157.5, 134.8, 129.6, 113.3, 82.3, 56, 55, 51, 42.6, 16.5; Anal. calcd for $\mathrm{C}_{13} \mathrm{H}_{18} \mathrm{O}_{4}$ : C, 65.53\%; H, 7.61\%. Found C, 65.50\%; H, 7.59\%.

Methyl-3-(4-chlorophenyl)-3-methoxy-2-methylpropanoate (35a/b). Diastereoisomer syn (35a): ${ }^{1} \mathrm{H}$ NMR (500MHz, $\left.\mathrm{CDCl}_{3}\right) \delta 1.15$ (d, $\left.J=6.5 \mathrm{~Hz}, 3 \mathrm{H}\right)$, 2.73-2.80 (m, 1H), 3.40 (s, 3H), 3.65 (s, 3H), 4.4 (d, $J=7.0 \mathrm{~Hz}, 1 \mathrm{H}), 7.19$ (d, $J=8.0 \mathrm{~Hz}$, $2 \mathrm{H}$ aromatics), 7.33 (d, $J=8.0 \mathrm{~Hz}, 2 \mathrm{H}$ aromatics); ${ }^{13} \mathrm{C} \mathrm{NMR}\left(125 \mathrm{MHz}, \mathrm{CDCl}_{3}\right) \delta 172.8$, 139.2, 132.6, 129, 127.9, 82.3, 56.6, 51, 36.9, 15; diastereoisomer anti (35b) ${ }^{1} \mathrm{H}$ NMR $\left(500 \mathrm{MHz}, \mathrm{CDCl}_{3}\right) \delta 1.15(\mathrm{~d}, J=6.54 \mathrm{~Hz}, 3 \mathrm{H}), 2.73-2.80(\mathrm{~m}, 1 \mathrm{H}), 3.40(\mathrm{~s}, 3 \mathrm{H}), 3.65$ (s, $3 \mathrm{H}), 4.41$ (d, $J=6.5 \mathrm{~Hz}, 1 \mathrm{H}), 7.19$ (d, $J=8.0 \mathrm{~Hz}, 2 \mathrm{H}$ aromatics), 7.33 (d, $J=8.0 \mathrm{~Hz}, 2 \mathrm{H}$ aromatics); $\left.{ }^{13} \mathrm{C} \mathrm{NMR} \mathrm{(125MHz,} \mathrm{CDCl}_{3}\right) \delta 174.8,139.3,129,127.9,82.4,56 ., 51,42.6$, 16.5; Anal. calcd for $\mathrm{C}_{12} \mathrm{H}_{15} \mathrm{ClO}_{3}$ : C, 59.39\%; H, 6.23\%. Found C, 59.39\%; H, 6.23\%.

Methyl 3-hydroxy-2-methylbutanoate (40a). Reaction time. $10 \mathrm{~h}$ [78\% of a single diastereomer (we were unable to isolate the anti diastereoisomer)]. ${ }^{1} \mathrm{H}$ NMR (300MHz, $\left.\mathrm{CDCl}_{3}\right) \delta 1.12\left(\mathrm{t}, J=6.5 \mathrm{~Hz}, 3 \mathrm{H}, \mathrm{CHCH}_{3}\right), 1.21$ (d, $J=6.42 \mathrm{~Hz}, 3 \mathrm{H}, \mathrm{C}_{3}$ terminal), 2.35$2.41(\mathrm{~m}, 1 \mathrm{H}), 3.66$ (s, 3H, OC$\underline{\mathrm{H}}_{3}$ ester), 3.74-3.81 (m, 1H, $\left.\mathrm{C} \underline{\mathrm{HOH}}\right) ;{ }^{13} \mathrm{C} \mathrm{NMR}$ $\left(75.4 \mathrm{MHz}, \mathrm{CDCl}_{3}\right) \delta 172.1,67,51,38.7,20.4,14.1$; Anal. calcd for $\mathrm{C}_{6} \mathrm{H}_{12} \mathrm{O}_{3}$ : C, 54.53\%; H, 9.15\%. Found C, 54.51\%; H, 9.12\%. 
Methyl 3-hydroxy-2-methylpentanoate (41a). Reaction time. 12 h; [82\% of a single diastereomer (we were unable to isolate the anti diastereoisomer)]. ${ }^{1} \mathrm{H} \mathrm{NMR} \mathrm{(300MHz}$, $\left.\mathrm{CDCl}_{3}\right) \delta 0.97\left(\mathrm{t}, 3 \mathrm{H}, \mathrm{CH}_{3}\right.$ terminal), $1.12\left(\mathrm{~d}, J=6.9 \mathrm{~Hz}, 3 \mathrm{H}, \mathrm{C}_{3} \mathrm{CH}\right.$ ), 1.58-1.64 (m, $2 \mathrm{H}, \mathrm{C}_{2} \mathrm{CH}_{3}$ ), 2.38-2.45 (m, $\mathrm{CH}_{3} \mathrm{C} \underline{\mathrm{HCO}}$ ), 3.66 (s, 3H, OC$\underline{H}_{3}$ ester), 3.70-3.75 (dt, $J=$ 5.9 and $5.5 \mathrm{~Hz}, 1 \mathrm{H}, \mathrm{C} \underline{\mathrm{HOH}}) ;{ }^{13} \mathrm{C} \mathrm{NMR}\left(75.4 \mathrm{MHz}, \mathrm{CDCl}_{3}\right) \delta 173.8(\mathrm{C}=\mathrm{O}), 69.8,51.2$, 38, 26.8, 14.3, 11.4. \%; Anal. calcd for $\mathrm{C}_{7} \mathrm{H}_{14} \mathrm{O}_{3}$ : C, 57.51\%; $\mathrm{H}, 9.65 \%$. Found C, $57.50 \%$; H, 9.63\%.

Methyl 3-hydroxy-2-methylhexanoate (42a). Reaction time. $10 \mathrm{~h}$; (76\% of a colorless oil, only a single diastereoisomer detected $\geq 95 \%) .{ }^{1} \mathrm{H}$ NMR $\left(\mathrm{C}_{6} \mathrm{D}_{6}, 300 \mathrm{MHz}\right) \delta 0.89(\mathrm{t}$, $3 \mathrm{H}, \mathrm{CH}_{3}$ terminal), $1.12(\mathrm{~d}, J=6.9 \mathrm{~Hz}, 3 \mathrm{H}), 1.5-1.8(\mathrm{~m}, 4 \mathrm{H}), 2.54(\mathrm{dq}, J=4.76$ and $6.9 \mathrm{~Hz}, 1 \mathrm{H}), 3.67(\mathrm{~s}, 3 \mathrm{H}), 4.0(\mathrm{dt}, J=4.0$ and $6.0 \mathrm{~Hz}, 1 \mathrm{H}, \mathrm{C} \underline{\mathrm{HOH}}) ;{ }^{13} \mathrm{C} \mathrm{NMR}\left(\mathrm{CDCl}_{3}\right.$, 75.4MHz) $\delta 174.6(\mathrm{C}=\mathrm{O}), 77.4,69.5,51,39.9,35.8,21.2,14.2$; Anal. calcd. for $\mathrm{C}_{8} \mathrm{H}_{16} \mathrm{O}_{3} \mathrm{C}, 59.97 \%$; H, 10.07\%; Found C, 59.97\%; H, 10.05\%.

Methyl 3-hydroxy-2-methylheptanoate (43a). Reaction time. $10 \mathrm{~h}$; (76\% of a fluid oil, only a single diastereoisomer detected $\geq 95 \%)$. $1 \mathrm{H} \mathrm{NMR}\left(300 \mathrm{MHz}, \mathrm{C}_{6} \mathrm{D}_{6}\right) \delta 0.85$ (t, $3 \mathrm{H}, \mathrm{CH}_{3}$ terminal), $1.23(\mathrm{~d}, J=7.3 \mathrm{~Hz}, 3 \mathrm{H}), 1.33-1.26(\mathrm{~m}, 2 \mathrm{H}), 1.59-1.49(\mathrm{~m}, 4 \mathrm{H}), 2.52$ $(\mathrm{dq}, J=4.4$ and $6.95 \mathrm{~Hz}, 1 \mathrm{H}), 3.44(\mathrm{~s}, 3 \mathrm{H}), 4.14$ (ddd, $J=1.8,4.3$ and $6.2 \mathrm{~Hz}, 1 \mathrm{H}$, $\mathrm{CHOH}) ;{ }^{13} \mathrm{C}$ NMR (75.4MHz, $\left.\mathrm{C}_{6} \mathrm{D}_{6}\right) \delta 174.6(\mathrm{C}=\mathrm{O}), 73.6,51.1,44.6,35.4,27.9,14.4$, 11.03; HRMS $\left(\mathrm{M}^{+}\right)$Calcd. for $\mathrm{C}_{7} \mathrm{H}_{14} \mathrm{O}_{3}$ 174.12559; Found 174.12550.

\section{Acknowledgements}

The authors thank Fapesp for a fellowship to CRM (n98/13424-1), CNPq for a fellowship to FC (301369/1987-9) and JCFG and SAE-Unicamp for a fellowship to LDF and Fapesp ( ${ }^{\circ}$ 98/14640-0 and 02/00461-3) and CNPq for financial support. We are grateful to Prof. Carol H. Collins for the English revision of this text.

\section{References}

1. For reviews see: (a) Drewes, S. E.; Roos, G. H. P. Tetrahedron 1988, 44, 4653. (b) Basavaiah, D.; Rao, P. D.; Hyma, R. S. Tetrahedron 1996, 52, 8001. (c) Ciganek, E., In Organic Reactions; John Wiley: New York, 1997; Vol. 51, Chapter 2, p 201. (d) Almeida, W. P.; Coelho, F. Quim. Nova 2000, 23, 98; Chem. Abstr. 2000, 132, 236532e. (e) Basavaiah, D.; Rao, A. J.; Satyanarayana, T. Chem. Rev. 2003, 103, 811.

2. (a) Basavaiah, D.; Sreenivasulu, B.; Reddy, R. M.; Muthukumaran, K. Synth. Commun. 2001, 31, 2987. (b) Yadav, J. S.; Reddy, B. V. S.; Madan, C. New J. 
Chem. 2001, 25, 1114. (c) Shanmugam, P.; Singh, P. R. Synlett 2001, 8, 1314. (d) Ravichandran, S. Synth. Commun. 2001, 31, 2055. (e) Shi, M.; Feng, Y. S. J. Org. Chem. 2001, 66, 406. (f) Micuch, P.; Fisera, L.; Cyranski, M. K.; Krygowski, T. M.; Krajcik, J. Tetrahedron 2000, 56, 5465. (g) Kataoka, T.; Kinoshita, H.; Kinoshita, S.; Iwamura, T.; Watanabe, S. Angew. Chem., Int. Ed. 2000, 39, 2358. (h) Micuch, P.; Fisera, L.; Cyranski, M. K.; Krygowski, T. M. Tetrahedron Lett. 1999, 40, 167. (i) Iwama, T.; Tsujiyama, S.; Kinoshita, H.; Kanematsu, K.; Tsurukami, Y.; Iwamura, T.; Watanabe, S.; Kataoka, T. Chem. Pharm. Bull. 1999, 47, 956. (j) Basavaiah, D.; Muthukumaran, K.; Sreenivasulu, B. Synlett 1999, 8, 1249. (k) Iwama, T.; Kinoshita, H.; Kataoka, T. Tetrahedron Lett. 1999, 40, 3741. (1) Kundu, M. K.; Mukherjee, S. B.; Balu, N.; Padmakumar, R.; Bhat, S. V. Synlett 1994, 6, 444. (m) Cablewski, T.; Faux, A. F.; Strauss, C. R. J. Org. Chem. 1994, 59, 3408.

3. For some examples of metal catalysis in Baylis-Hillman reaction, see: (a) Shi, M.; Jiang, J. K.; Cui, S. C. Tetrahedron 2001, 57, 7343. (b) Akiyama, H.; Fujimoto, T.; Ohshima, K.; Hoshino, K.; Saito, Y.; Okamoto, A.; Yamamoto, I.; Kakehi, A.; Iriye, R. Eur. J. Org. Chem. 2001, 12, 2265. (c) Comins, D. L.; Hiebel, A. C.; Huang, S. L. Org. Lett. 2001, 3, 769. (d) Shi, M.; Feng, Y. S. J. Org. Chem. 2001, 66, 406. (e) Kataoka, T.; Kinoshita, H.; Kinoshita, S.; Iwamura, T.; Watanabe, S. Angew. Chem., Int. Ed. 2000, 39, 2358. (f) Akiyama, H.; Fujimoto, T.; Ohshima, K.; Hoshino, K.; Yamamoto, I.; Iriye, R. Org. Lett. 1999, 1, 7447. (g) Aggarwal, V. K.; Mereu, A.; Tarver, G. J.; McCague, R. J. Org. Chem. 1998, 63, 7183.

4. (a) Rosa, J. N.; Afonso, C. A. M.; Santos, A. G. Tetrahedron 2001, 57, 4189. (b) Kitazume, T.; Tamura, K.; Jiang, Z. J.; Miyake, N. Kawasaki, I. J. Fluorine Chem. 2002, 115, 49. (c) Aggarwal, V. K.; Emme, I.; Mereu, A. Chem. Commun. 2002, 15, 1612. (d) Kim, E.J.; Ko, S. Y.; Song, C.E. Helvetica Chim. Acta 2003, 86, 894. (e) Mateus, N.M.M.; Branco L. C.; Lourenço N. M. T.; Afonso, C. A. M. Green Chemistry 2003, 5, 347. (f) Kabalka, G.W.; Venkataiah B.; Dong, G. Tetrahedron Lett. 2003, 44, 4673.

5. (a) Yu, C. Z.; Liu, B.; Hu, L. Q. J. Org. Chem. 2001, 66, 5413. (b) Auge', J.; Lubin, N.; Lubineau, A. Tetrahedron Lett. 1994, 35, 7947.

6. (a) Pei, W.; Wei, H. X.; Li, G. G. Chem. Commun. 2002, 20, 1856 and references cited therein. (b) Li, G. G.; Wei, H. X.; Gao, J. J.; Caputo, T. D. Tetrahedron Lett. 2001, 41, 1.

7. (a) Almeida, W. P.; Coelho, F. Tetrahedron Lett. 1998, 39, 8609. (b) Coelho, F.; Almeida, W. P.; Veronese, D.; Mateus, C. R.; Lopes, E. C. S.; Silveira, G. P. C.; Rossi, R. C.; Pavam, C. H. Tetrahedron 2002, 58, 7437.

8. Coelho, F.; Almeida, W. P.; Mateus, C. R.; Feltrin, M.; Costa, A. M. Tetrahedron 2001, 57, 6901.

9. Brown, J. M.; Cutting, I. J. Chem. Soc., Chem. Commun. 1985, 578. 
10. Brown, J. M. Angew. Chem., Int. Ed. 1987, 26, 190.

11. Brown, J. M.; Cutting, I.; James, A. P. Bull. Soc. Chim. Fr. 1988, 211.

12. Yamamoto, K.; Takagi, M.; Tsuji, J. Bull. Chem. Soc. Jpn. 1988, 319.

13. Brown, J. M.; Rose, M.; Knight, F. I.; Wienand, A. Recl. Trav. Chim. Pays-Bas 1995, 114, 247.

14. Brown, J. M.; Evans, P. L.; James, A. P. Org. Synth. 1990, 68, 64.

15. Farington, E.; Franchini, M.C.; Brown, J. M. Chem.Commun. 1998, 277.

16. Sato, S.; Matsuda, I.; Shibata, M. J. Organomet. Chem. 1989, 377, 347.

17. Almeida, W. P.; Mateus, C. R.; Coelho, F. Tetrahedron Lett. 2000, 41, 2533.

18. Heathcock, C.H., In Asymmetric Synthesis, Morrison, J.D., Ed.; Academic Press: London, 1984; Vol. 3, part B, p 115.

19. Bouzide, A. Org. Lett. 2002, 4, 1347.

20. Jackson, R.F.W.; Standen, S.P.; Clegg, W.; McCamley, A. Tetrahedron Lett. 1992, 33, 6197 and references cited therein.

21. (a) Hoffmann, R.W. Chem. Rev. 1989, 89, 1841; (b) Kahn, S.D.; Pau, C.F.; Chamberlin, A.R.; Hehre, W. J. J. Am. Chem. Soc. 1987, 109, 650 and references cited therein.

22. (a) Morita, K; Suzuki, K.; Hirose, H. Bull. Soc. Chem. Jpn. 1968, 41, 2815. (b) Lu, X.Y.; Zhang, C.M.; Xu, Z.R. Acc. Chem. Res. 2001, 34, 535. (c) Hayase, T.; Shibata, T.; Soai, K.; Wakatsuki,Y. Chem. Commun. 1998, 12, 1271. 